\title{
FUTURES MARKET DEVELOPMENT AND ECONOMIC GROWTH
}

\author{
İlkay Şendeniz-Yüncü* \\ Levent Akdeniz $^{* *}$ \\ Kürşat Aydoğan ${ }^{* * *}$
}

February 2007

\begin{abstract}
This paper investigates the relationship between futures market development and economic growth using both dynamic panel and time-series methods in developed and emerging markets. The dynamic panel estimation results suggest that futures market development has a significant impact on economic growth. This finding is further supported by time-series analysis for individual countries in our data set.
\end{abstract}

Keywords: Futures market; economic growth; dynamic panel; time-series.

JEL Classification: C22; C23; E44; G10

\footnotetext{
* Corresponding author. Faculty of Business Administration, Bilkent University, Bilkent, 06800, Ankara Turkey.

E-mail address: sendeniz@bilkent.edu.tr. Tel: +90 312290 1508, +90 312290 1928; Fax: +90 312 2664958.

** Faculty of Business Administration, Bilkent University, Bilkent, 06800, Ankara Turkey. E-mail address: akdeniz@bilkent.edu.tr

*** Faculty of Business Administration, Bilkent University, Bilkent, 06800, Ankara Turkey. E-mail address: aydogan@bilkent.edu.tr
} 


\section{Introduction}

Several studies have shown that there is a positive relationship between a country's economic growth and the development of its financial market ${ }^{1}$. It is intuitive that well-developed financial intermediaries in a country with a well-functioning financial market increase the efficiency with which a greater amount of capital accumulation is facilitated and a greater amount of funds are allocated to profitable investments. However, researchers have not yet thoroughly investigated the underlying mechanisms that suggest a positive relationship between the degree of development of the financial system and economic growth. For instance, does the development of derivative contracts contribute to economic growth?

One major function of financial markets is to reallocate risk between different economic agents. On the one hand, reallocation of risk enables borrowers to tailor their risk portfolios and therefore, to achieve greater access to capital. On the other hand, savers become better able to diversify their risk and make more funds available for borrowing. As a result, an economy unquestionably gains from this efficient capital allocation generated from improved risk sharing. In a financial system, innovations such as derivatives are viewed as mechanisms to allocate capital efficiently and to share risk.

Derivatives markets are an integral part of a financial system. They allow markets to provide information about market clearing price, which is an essential component of an efficient economic system. In particular, futures markets widely distribute equilibrium prices that reflect demand and supply conditions, and knowledge of those prices is essential for investors, consumers, and producers to make informed decisions. As a result, investments become more productive and lead to a higher rate of

\footnotetext{
${ }^{1}$ See Levine (2005) for a full review of this research.
} 
economic growth. In addition, derivative instruments provide an opportunity for hedging risk, which in turn, leads to economic growth.

There have been numerous theoretical studies that demonstrate the link between financial risk and economic growth. Acemoglu and Zilibotti (1997) show that reducing financial risk through holding diversified portfolios allow agents to invest in high-return projects with a positive influence on growth. Angeletos and Calvet (2006) illustrate that improvements in entrepreneurial risk sharing through the introduction of new hedging instruments will have a positive effect on savings and medium run growth. Moreover, Turnovsky and Bianconi (2005) and Storesletten et al. (2004) show that idiosyncratic risks play an important role in aggregate risk; thus, reducing the idiosyncratic risks of economic agents leads to economic growth. Krebs (2002) also shows that a reduction in the variation in firm specific risk decreases the ratio of physical to human capital and increases the total investment return and growth.

On the empirical side, there are various studies that investigate the relationship between financial development and economic growth. Among many others, King and Levine (1993), Beck et al. (2000), Levine et al. (2000), Jeong et al. (2003), and Şendeniz-Yüncü et al. (2007) examine the relationship between financial intermediary development, namely banking sector development, and economic growth. Moreover, Atje and Jovanovic (1993), Demirgüç-Kunt and Levine (1996a,b), Harris (1997), Levine and Zervos (1998), Rousseau and Wachtel (1998), and Arestis et al. (2001) study the relationship between stock market development and economic growth. Beck and Levine (2004) investigate the impact of both stock markets and banks on economic growth applying generalized-method-of moments techniques developed for dynamic panels. As a result of these studies, it is now a common belief that well-functioning 
financial intermediaries and markets ameliorate information and transaction costs to promote efficient resource allocation, and, hence, economic growth. However, researchers have not thoroughly examined the underlying mechanisms that lead to the positive relationship between the degree of development of the financial system and economic growth. Although the relationship between the banking sector, stock market and economic growth is extensively examined in the literature, there is no study that focuses on the effect of the development of derivative markets on economic growth. For instance, is it only the banking sector or also the stock market within the financial system that contributes to economic growth? Does the development of futures markets contribute to economic growth as well?

In this study, we use both dynamic panel and time-series approaches to examine the relationship between futures market development and economic growth in developing and developed countries. Analyzing this relationship is important, because clarifying the role of futures markets in economic growth may lead to government policies that support developing their derivative markets in order to promote economic growth. Especially in emerging markets, most of the production takes place in privately held small firms where risk sharing is absent most of the time. Thus, promoting financial markets and services that ease risk sharing in these countries may result in welfare increase.

The organization of the rest of the paper is as follows. Section 2 describes the data; the Dynamic Panel Model and its results are discussed in section 3; Section 4 contains time-series analyses and their results; and section 5 concludes the paper. 


\section{Data}

In this study, we perform both dynamic panel and time-series analyses in order to examine the relationship between futures market development and economic growth for both developed and developing countries. In the panel data analysis, we include 15 countries with annual observations in the period 1994-2003. For the time-series analyses, we use quarterly data for 22 countries, 17 of which are developed and 5 of which are emerging markets, for the period 1982:Q3-2005:Q3. We report the countries and time periods included in the study in Table 1.

$$
<<\text { Table } 1 \text { here }>>
$$

We use the total value of stock index futures contracts to nominal Gross Domestic Product (GDP) ratio to proxy the futures market development for each country in our data set. We obtain the total value of stock index futures contracts by multiplying the volume of contracts traded by contract prices in each period. We acquire the futures data from Datastream Advance 3.5. As for the economic growth indicator, we use logarithm of seasonally adjusted real GDP, which is obtained from the International Monetary Fund's International Financial Statistics Online Service. All data points are in the countries' national currencies. We present the ratio of yearly values of stock index futures contracts to the GDP for each country in Table 2.

$$
<<\text { Table } 2 \text { here }>>
$$

In order to assess the strength of the independent link between futures market development and economic growth, control for other potential determinants of economic growth is needed in the analyses. For this purpose we use banking sector development, inflation rate, and Foreign Direct Investment (FDI) as a percentage of GDP as control variables. Consistent with the literature, we measure the banking sector 
development as the volume of credit extended to the private sector by deposit money banks divided by GDP. These are commonly used control variables in the literature ${ }^{2}$. We also use dummy variables to control for the effect of financial crisis. The dummy variable takes a value of one during the years of crises and in the preceding and following years, and zero in the other years.

\section{Dynamic Panel Model}

To examine the relationship between futures market development and economic growth, we employ the generalized-method-of moments (GMM) estimators developed for dynamic panel models by Arellano and Bond (1991) and Holtz-Eakin et al. (1988). As Edison et al. (2002) argue, the dynamic panel approach offers many advantages for researchers in the estimation of a relationship. First, dynamic panel econometric technique allows us to exploit the time-series nature of the relationship between the variables with pooled cross-section and time-series data. Second, by using this technique we are able to remove any bias created by unobserved country-specific effects. Third, it controls for the potential endogeneity of all explanatory variables. Thus, we view the dynamic panel estimator as a better technique to examine the relationship mentioned above.

The data used in dynamic panel estimation covers the period 1994-2003. Table 3 presents descriptive statistics and correlations. Belgium had the highest growth rate of $10.17 \%$ in 1999 , while Hong Kong, China had the lowest growth rate of $-5.45 \%$ in 1998. In terms of the value of futures contracts as a percentage of GDP, Hong Kong,

\footnotetext{
${ }^{2}$ See Edison et al. (2002) and Beck and Levine (2004).
} 
China had the highest value of $291 \%$ in 1997 . Belgium, with only $2 \%$, had the lowest value of futures contracts as a percentage of GDP in 1994.

$<<$ Table 3 here $>>$

We start with the following regression equation:

$$
y_{i, t}-y_{i, t-1}=(\alpha-1) y_{i, t-1}+\beta^{\prime} X_{i, t}+\eta_{i}+\varepsilon_{i, t}
$$

where $y$ is the logarithm of real GDP, $X$ represents the set of explanatory variables including the futures market development measure, $\eta$ is an unobserved country-specific effect, $\varepsilon$ is the error term, and the subscripts $i$ and $t$ represent country and time period, respectively.

The above equation can be rewritten as

$$
y_{i, t}=\alpha y_{i, t-1}+\beta^{\prime} X_{i, t}+\eta_{i}+\varepsilon_{i, t}
$$

Arellano and Bond (1991) propose to difference the above equation in order to eliminate the country-specific effect:

$$
y_{i, t}-y_{i, t-1}=\alpha\left(y_{i, t-1}-y_{i, t-2}\right)+\beta^{\prime}\left(X_{i, t}-X_{i, t-1}\right)+\left(\varepsilon_{i, t}-\varepsilon_{i, t-1}\right)
$$

Differencing eliminates the country-specific effect; however, it introduces a new econometric problem. The new error term in the difference equation $\left(\varepsilon_{i, t}-\varepsilon_{i, t-1}\right)$ is now correlated with the lagged dependent variable $\left(y_{i, t-1}-y_{i, t-2}\right)$. By assuming that the error term is not serially correlated, and the explanatory variables are weakly exogenous (i.e., the explanatory variables are uncorrelated with future error terms), the GMM dynamic panel estimator uses the following moment conditions:

$$
\begin{aligned}
& E\left[y_{i, t-s}\left(\varepsilon_{i, t}-\varepsilon_{i, t-1}\right)\right]=0 \text { for } s \geq 2 ; t=3, \ldots, T, \\
& E\left[X_{i, t-s}\left(\varepsilon_{i, t}-\varepsilon_{i, t-1}\right)\right]=0 \text { for } s \geq 2 ; t=3, \ldots, T,
\end{aligned}
$$


Arellano and Bond (1991) suggest a two-step GMM estimator using the above moment conditions. In the first step, the error terms are assumed to be independent and homoskedastic across countries and over time. In the second step, the residuals from the first step are used to construct a consistent estimate of the variance-covariance matrix. The consistency of the GMM estimator mainly depends on the assumptions that the error terms do not exhibit second order serial correlation and that the instruments are valid. To check whether or not these assumptions hold, we run the Sargan and serial correlation tests. Failure to reject the null hypothesis of these tests implies that the assumptions of the estimation hold.

\subsection{Results of Dynamic Panel Analyses}

In this section, we present the results of the GMM dynamic panel estimation. As can be seen in Table 4, the development of futures markets has a positive significant effect on economic growth.

$<<$ Table 4 here $>>$

The results in Table 4 are intuitive. One the one hand, well-functioning futures markets allow for greater and more efficient risk sharing, thereby making it possible for firms to undertake relatively riskier projects and, hence, promote growth. On the other hand, futures markets widely distribute equilibrium prices that reflect demand and supply conditions, and knowledge of those prices allows investors, consumers, and producers to make informed decisions. Consequently, the amelioration of information and transaction costs fosters efficient resource allocation, thus leading to economic growth.

Moreover, our panel study results further show that while Foreign Direct Investment (FDI) has a statistically significant positive effect on economic growth, 
inflation and economic crises have statistically significant negative effects on economic growth, as expected. In the regression, our banking sector development variable (as it is commonly measured in the literature as the volume of credit extended to the private sector by deposit money banks, divided by GDP) enters statistically insignificantly with a positive sign. This result is not surprising, because Beck and Levine (2004) also report that the effect of banking sector development becomes statistically insignificant when the regression is controlled for inflation and trade openness. Furthermore, our specification tests indicate that we cannot reject the null hypothesis of no second order serial correlation in the differenced error term and that our instruments are valid.

\section{Time-series Approach}

Exploring the time-series properties of the futures market development and the economic growth relationship for individual countries can be further informative. Time series techniques allow us to investigate this relationship in further detail for individual countries through time. In order to examine the relationship between futures market development and economic growth through time for an individual country, we run the following time-series tests using quarterly data: cointegration tests to see the comovement of variables in the long run and to select a vector error correction model (VECM), causality tests to analyze the direction of causalities, variance decompositions to break down the variance of the forecast error for each variable into components, and the impulse-response function to trace the effect of a one-time shock to one of the endogenous variables on current and future values of itself and the other endogenous variables. 


\subsection{Unit Root Tests}

Existence of a stationary linear combination between non-stationary series suggests the existence of a cointegrating relationship between them. Therefore, the stationarity of the series should be tested. Augmented-Dickey-Fuller (ADF) and Phillips-Perron (PP) unit root tests are used to determine the stationarity of the value of futures market contracts as a percentage of GDP and logarithm of seasonally adjusted real GDP series. The quarterly value of futures market contracts divided by quarterly GDP data is represented with the name "FUTURES", and the seasonally adjusted quarterly real GDP data is represented with the name "GDP". Both tests have the null hypothesis of the existence of a unit root, rejection of which indicates stationarity.

At least one test failed to reject the null hypothesis at the 5 percent significance level for 20 series in levels, indicating non-stationarity. For the other 2 countries, Kwiatkowski-Phillips-Schmidt-Shin (KPSS) test results showed non-stationarity of the series in levels. At least one test rejected the null hypothesis at the 5 percent significance level for 19 series in first differences in the sample ${ }^{3}$. For the other 3 countries, KPSS test results showed stationarity of the first differenced series. KPSS test was also performed for the series that showed inconsistency between the ADF and PP tests. KPSS test results confirmed stationarity of the first differenced series. Table 5 presents the results of the ADF and PP unit root tests for 22 countries in levels and first differences.

$<<$ Table 5 here $>>$

\footnotetext{
${ }^{3}$ Lags between 1 and 10 were checked, and the lag that minimized the Akaike Information Criterion (AIC) was chosen when performing the ADF test. The Newey-West bandwidth automatic selection was used when performing the PP unit root test.
} 


\subsection{Cointegration Tests}

Although individual series are non-stationary, a linear combination of these series may be stationary. Therefore, such a stationary linear combination, i.e., the cointegrating equation, is searched. Johansen's cointegration tests are performed to see if the non-stationary series FUTURES and GDP move together over time and if cointegration exists among them. Table 6 presents the results of the Johansen cointegration tests.

$<<$ Table 6 here $>>$

The finding of cointegration between the series FUTURES and GDP for all countries suggests the presence of co-movements among the variables, indicating longrun stationarity. To see the speed of adjustments of the variables to deviations from their common stochastic trend, the error correction model (ECM) is used. The ECM corrects the deviation from the long-run equilibrium by short-run adjustments.

\subsection{Granger-causality Tests}

Granger-causality tests are performed to see the direction of the causal link between futures market development and economic growth. Granger-causality establishes the leading role of one variable in the fluctuations of another. The basic rationale of Granger-causality is that the change in FUTURES Granger-causes the change in GDP, if past values of the change in FUTURES improve unbiased leastsquares predictions about the change in GDP. We run Granger-causality regressions of the following form:

$$
\Delta G D P_{t}=\sum_{i=1}^{n} \pi_{11} \Delta G D P_{t-i}+\sum_{i=1}^{n} \pi_{12} \Delta F U T U R E S_{t-i}+u_{t}
$$




$$
\triangle F U T U R E S_{t}=\sum_{i=1}^{n} \pi_{22} \Delta F U T U R E S_{t-i}+\sum_{i=1}^{n} \pi_{21} \Delta G D P_{t-i}+v_{t}
$$

where $\Delta$ is the change operator, and $u$, and $v$ are the error terms. For the right-hand side of the above equations, we tried lags between 1 and 10 and chose the lag that yielded the smallest Akaike Information Criteria (AIC).

In the above Granger-causality regressions, if $\pi_{12}$ parameters are jointly zero, it is indicated that futures market development does not Granger-cause GDP growth, whereas if $\pi_{21}$ parameters are jointly zero, it is indicated that GDP growth does not Granger-cause futures market development. The null hypotheses are that futures market development does not Granger-cause GDP growth in the first regression and that GDP growth does not Granger-cause futures market development in the second regression. If none of the null hypotheses are rejected, it can be concluded that GDP growth and futures market development are Granger-independent. If both are rejected, it implies a Granger-feedback between GDP growth and futures market development.

Table 7 presents the results of Granger-causality tests. It is observed that in Brazil, France, Japan and Portugal the change in FUTURES Granger-causes the change in GDP $(\Delta$ FUTURES $\Rightarrow \Delta$ GDP). The reverse causality is observed in Hong Kong, China, the Netherlands, Norway and Portugal; that is, the change in GDP Grangercauses the change in FUTURES ( $\Delta$ GDP $\Rightarrow \Delta$ FUTURES). The bi-directional causality in Portugal suggests interdependence between the futures market development and economic growth. The causalities from FUTURES to GDP are observed with a 5 percent significance level for Brazil, France, Japan and Portugal; the causalities from GDP to FUTURES are observed with a 5 percent significance level for Hong Kong, 
China, Norway and Portugal, and with a 10 percent significance level for the Netherlands. This relationship is not observed for the other countries.

$<<$ Table 7 here $>>$

\subsection{Vector Error Correction Models}

Engle and Granger (1987) argued that in the presence of cointegration, there will be a corresponding error-correction representation. Here, the existence of cointegration between FUTURES and GDP series leads us to use the Vector Error Correction Model (VECM). A cointegration in variables indicates long-run stationarity, but gives no information about the speed of adjustments of the variables to deviations from their common stochastic trend. The VECM should be used to correct the deviation from the long-run equilibrium by short-run adjustments. The VECM is constructed by adding an adjustment parameter to the cointegration equation. Consequently, short-run adjustments correct deviations from the long-run equilibrium. VECM will be used in the calculation of variance decomposition and impulse-response function among FUTURES and GDP series.

The following VECM is constructed. The fourth component of each equation is the error correction term (ECT) that is formed with the cointegrating vector. The sign and the size of the coefficient of the ECT in each equation reflect the direction and speed of adjustments in the dependent variable to deviations from the linear long-run relationship.

The VECM has the following form:

$$
\begin{aligned}
\Delta \text { FUTURES }_{t}= & d_{1}+a_{11}(L) \Delta F U T U R E S_{t-1}+a_{12}(L) \Delta G D P_{t-1}+ \\
& g_{1}\left(\text { FUTURES }_{t-1}+b_{12} \text { GDP }_{t-1}+c_{0}\right)+\varepsilon_{1 t}
\end{aligned}
$$




$$
\begin{aligned}
\Delta G D P_{t}= & d_{2}+a_{21}(L) \Delta F U T U R E S_{t-1}+a_{22}(L) \Delta G D P_{t-1}+ \\
& g_{2}\left(\text { FUTURES } S_{t-1}+b_{12} G D P_{t-1}+c_{0}\right)+\varepsilon_{2 t}
\end{aligned}
$$

where $\Delta$ is the change operator; $d_{1}, d_{2}$, and $c_{0}$ are constants; $L$ is the lag operator $\left[a_{11}(L): a_{11.0} L^{0}+a_{11.1} L^{1}+\ldots(\right.$ a polynomial in $\left.\mathrm{L})\right] ; g_{1}$, and $g_{2}$ are the adjustment parameters; and $b_{12}$ is the cointegration coefficient. Adjustment parameters and the coefficients of cointegration equations for each country are given in Table 8 .

$<<$ Table 8 here $>>$

\subsection{Variance Decomposition and Impulse-Response Function Analysis}

We performed variance decomposition and impulse-response function (IRF) analyses to see the relationship between futures market development and economic growth. Variance decomposition breaks down the variance of the forecast error for each variable into components that can be attributed to each of the endogenous variables. Thus, it provides information about the relative importance of each random innovation in how it affects the variables.

Table 9 shows the variance decompositions of FUTURES and GDP series for 22 countries. To illustrate the interpretation of Table 9, for Belgium, FUTURES innovations cause $100 \%$ of the variation in its forecast error in the first period, while GDP innovations cause zero percent of the same variation. In the variance decomposition of GDP, GDP innovations cause $81.26 \%$ of the variation in their forecast error in the first period, while FUTURES innovations cause $18.74 \%$ percent of the same variation. Looking at the whole 10 periods, FUTURES innovation yields much greater variation in GDP forecast error $(18.74 \%-30.65 \%)$ relative to the variation that GDP innovation yields in FUTURES (0\% - 2.21\%). In other words, FUTURES plays a much more important role in explaining the variation in GDP. The countries other than 
Belgium, in which the variance decomposition analysis results support the view that futures markets affect economic growth, are Brazil, France, Hungary, Italy and Portugal. FUTURES innovation yields high variation in GDP forecast error for Brazil $(19.12 \%-44.70 \%)$, France $(44.17 \%-75.67 \%)$, Hungary $(38.50 \%-63.56 \%)$, Italy $(32.21 \%-42.35 \%)$ and Portugal $(18.61 \%-32.67 \%)$.

$$
<<\text { Table } 9 \text { here }>>
$$

An impulse-response function traces the effect of a one-time shock to an endogenous variable on current and future values of itself and of the other endogenous variables. Impulse-response function analysis shows the response of an endogenous variable $i$ at time $t+s$ to the changes in errors of the other variable $j$ at time $t$. More specifically,

$$
\psi_{s}=\frac{\partial y_{i, t+s}}{\partial \varepsilon_{j t}}
$$

where $y$ is the vector of endogenous variables, and $\varepsilon$ is the vector of the error term. Estimates of the impulse-response function analysis are shown in Table 10. It is observed that, in general, one standard deviation FUTURES innovation affects GDP in the long term.

$$
<<\text { Table } 10 \text { here }>>
$$

The above variance decomposition and impulse-response function analyses are factorized by Cholesky Decomposition, and ordering for Cholesky is FUTURES to GDP. If the residuals of FUTURES and GDP are uncorrelated, i.e., less than 0.2, a change in the ordering does not affect the results. However, if the correlation coefficients of the residuals are greater than 0.2 , ordering is important. After checking for the residual correlation matrices, it is seen that for Australia, Austria, Belgium, 
France, Hungary, Italy, New Zealand and Portugal the residuals of FUTURES and GDP are correlated, i.e., correlation coefficients are greater than 0.2 . This makes the ordering important for these countries. Therefore, for these eight countries, the variance decomposition and impulse-response function analyses are repeated for the reverse ordering, i.e., GDP to FUTURES. The results are given in Table 11 for variance decomposition analysis. It is seen that FUTURES innovation yields high variation in GDP forecast error for France (18.32\% - 25.75\%), Hungary $(14.42 \%-27.14 \%)$, New Zealand $(19.77 \%-29.05 \%)$ and Portugal $(17.10 \%-32.94 \%)$ with one period lag. To summarize, variance decomposition analyses results support the existence of a relationship between futures market development and economic growth in Brazil, France, Hungary and Portugal, taking Cholesky ordering into consideration.

$$
<<\text { Table } 11 \text { here }>>
$$

Estimates of the impulse-response function analysis for the reverse ordering are shown in Table 12. The results suggest a relationship between futures markets development and economic growth in Belgium, France and Hungary in the long term.

$$
<<\text { Table } 12 \text { here }>>
$$

\section{Conclusion}

Our dynamic panel study results show that there exists a statistically significant positive relationship between futures market development and economic growth. Because the dynamic panel estimator controls for the potential endogeneity of all explanatory variables and un-observed country specific effects, our results are not due to such possible biases. Our panel study results are consistent with models that predict that well-functioning financial markets provide opportunities for firms to have more 
efficient and greater risk sharing along with amelioration of information and transaction costs, and thereby, promote economic growth.

Moreover, it is appealing to know if futures market development over time within a country has an effect on economic growth. Time-series properties of the relationship between futures market development and economic growth yield mixed results. Our Granger-causality test results show that in Brazil, France, Japan and Portugal, futures market development Granger-causes economic growth. Variance decomposition analysis results also support the existence of a relationship between futures market development and economic growth in Brazil, France, Hungary and Portugal. In general, these are the countries that have medium-sized yearly futures market values relative to their GDPs in our dataset. However, notably, we do not observe a significant impact of futures market development on economic growth for countries like Australia, Germany, Hong Kong, China, the Netherlands, Sweden, Switzerland, the United Kingdom and the United States that have large futures market values relative to their GDPs or for countries like Austria, Belgium, Canada, Italy, New Zealand and Spain that have small futures market values relative to their GDPs. The results of the impulse-response function analysis suggest that one-time shock to futures markets affects GDP in the long term.

To summarize, our panel data estimations suggest that futures market development has a significant impact on economic growth, whereas our time-series estimations in general indicate that this relationship is more robust for the countries that have medium-sized futures market value relative to their GDPs. 


\section{REFERENCES}

Acemoglu, D., Zilibotti F., 1997. Was prometheus unbound by chance? Risk, diversification, and growth. Journal of Political Economy 105, 709-751.

Angeletos, G., Calvet, L.E., 2006. Idiosyncratic production risk, growth and the business cycle. Journal of Monetary Economics 53, 1095-1115.

Arellano, M., Bond S., 1991. Some tests of specification for panel data: Monte Carlo evidence and an application to employment equations. Review of Economic Studies $58,277-297$.

Arestis, P., Demetriades P.O., Luintel K.B., 2001. Financial development and economic growth: the role of stock markets. Journal of Money, Credit and Banking 33(1), 1641.

Atje, R., Jovanovic, B., 1993. Stock markets and development. European Economic Review 37, 632-640.

Beck, T., Levine, R., Loayza, N., 2000. Finance and the sources of growth. Journal of Financial Economics 58(1), 261-300.

Beck, T., Levine, R., 2004. Stock markets, banks, and growth: Panel evidence. Journal of Banking and Finance 28, 423-442.

Demirgüç-Kunt, A., Levine, R., 1996a. Stock markets corporate finance and economic growth. World Bank Economic Review 10(2), 223-240.

Demirgüç-Kunt, A., Levine, R., 1996b. Stock market development and financial intermediaries: stylized facts. World Bank Economic Review 10(2), 291-322.

Edison, H.J., Levine, R., Ricci, L., Slok, T., 2002. International financial integration and economic growth. Journal of International Money and Finance 21, 749-776. 
Engle, R.F., Granger, C.W.J., 1987. Co-integration and error correction: representation, estimation and testing. Econometrica 55, 251-276.

Harris, R.D.F., 1997. Stock markets and development: A re-assessment. European Economic Review 41, 139-146.

Holtz-Eakin, D., Newey, W., Rosen, H.S., 1988. Estimating vector autoregressions with panel data. Econometrica 56 (6), 1371-1395.

Jeong, W., Kymn, K.O., Kymn, C.J., 2003. The long-run interdependence of bankhealth, investment-oriented bank loans, and economic performance: A time-series analysis. The Quarterly Review of Economics and Finance 43, 11-30.

King, R.G., Levine, R., 1993. Finance and growth: Schumpeter might be right. Quarterly Journal of Economics 108, 717-38.

Krebs, T., 2002. Growth and welfare effects of business cycles in economies with idiosyncratic human capital risk. Review of Economic Dynamics 6, 846-868.

Levine, R., 2005. Finance and growth: Theory and evidence, in: Aghion, P., Durlauf, S. (Eds.), Handbook of Economic Growth, Vol. 1. Part 1. North-Holland Elsevier Publishers, Amsterdam, pp. 865-934.

Levine, R., Loayza, N., Beck, T., 2000. Financial intermediation and growth: Causality and causes. Journal of Monetary Economics 46, 31-77.

Levine, R., Zervos, S., 1998. Stock markets, banks, and economic growth. The American Economic Review 88(3), 537-558.

Rousseau, P.L., Wachtel, P., 1998. Financial intermediation and economic performance: Historical evidence from five industrial countries. Journal of Money, Credit and Banking 30, 657-678. 
Şendeniz-Yüncü, İ., Akdeniz, L., Aydoğan, K., 2007. Interdependence of the banking sector and the real sector: Evidence from OECD countries. Applied Economics, forthcoming.

Storesletten, K., Telmer, C.I., Yaron, A., 2004. Cyclical dynamics in idiosyncratic labor-market risk. Journal of Political Economy 112, 695-717.

Turnovsky, S.J., Bianconi, M., 2005. The welfare gains from stabilization in a stochastically growing economy with idiosyncratic shocks and flexible labor supply. Macroeconomic Dynamics 9, 321-357. 
Table 1. Countries in the sample

\begin{tabular}{|c|c|c|c|}
\hline $\begin{array}{l}\text { Country } \\
\text { Names }\end{array}$ & Futures Exchanges & Index Name & Period \\
\hline \multicolumn{4}{|c|}{ Developed Markets } \\
\hline Australia* & Sydney Futures Exchange (SFE) & SPI 200 & 2000:III-2005:III \\
\hline Austria & Wiener Börse (OTOB) & ATX & 1992:IV-2005:III \\
\hline Belgium & Belgian Futures \& Options Exchange (BELFOX) & BEL20 & 1994:I-2005:III \\
\hline Canada* & Toronto Futures Exchange (TFE) & TORONTO 35 & 1991:II-2000:I \\
\hline Denmark & $\begin{array}{l}\text { Guarantee Fund for Danish Options and Futures } \\
\text { (FUTOP) }\end{array}$ & $\mathrm{OMXC} 20$ & 1992:III-2005:III \\
\hline France* & Marche a Terme International de France (MATIF) & MONEP - CAC 40 & 1999:II-2005:III \\
\hline Germany & Deutsche Terminboerse Gmbh (DTB) & EUREX - DAX & 1991:I-2005:III \\
\hline Italy* & Mercato Italiano Futures (MIF) & MIB 30 & 1995:I-2004:IV \\
\hline Japan & Osaka Stock Exchange (OSE) & OSX - NIKKEI 225 & 1988:IV-2005:III \\
\hline Netherlands & AEX - Optiebeurs (AEX) & AEX & 1989:I-2005:III \\
\hline New Zealand* & The NZ Futures \& Options Exchange (NZFOE) & NZFE - NZSE 10 & 1995:IV-2003:III \\
\hline Norway & Oslo Stock Exchange (OSLO) & OBX & 1992:IV-2005:III \\
\hline Spain & Mercado De Futuros Financieros (MEFF) & IBEX 35 PLUS & 1992:III-2005:III \\
\hline Sweden & The OMLX Exchange (OMLX) & OMXS30 & 1990:II-2005:III \\
\hline Switzerland & $\begin{array}{l}\text { Swiss Options \& Financial Futures Exchange } \\
\text { (SOFFEX) }\end{array}$ & EUREX - SMI & 1991:I-2005:III \\
\hline United & London International Financial Futures \& Options & FTSE 100 & 1984:III-2005:III \\
\hline Kingdom & Exchange (LIFFE) & & \\
\hline United States & Chicago Mercantile Exchange (CME) & S\&P 500 & 1982:III-2005:III \\
\hline \multicolumn{4}{|c|}{ Emerging Markets } \\
\hline Brazil & Bolsa de Mercadorias \& Futuros (BM\&F) & BOVESPA & 1994:I-2005:III \\
\hline $\begin{array}{l}\text { Hong Kong, } \\
\text { China }\end{array}$ & Hong Kong Futures Exchange (HKFE) & HANG SENG & 1988:II-2005:III \\
\hline Hungary* & Budapest Stock Exchange (BSE) & BUX & 1995:II-2005:III \\
\hline Portugal* & Bolsa de Derivados de Porto (BDP) & PSI 20 & 1996:III-2005:III \\
\hline South Africa & South African Futures Exchange (SAFEX) & ALL SHARE 40 & 1990:III-2005:III \\
\hline
\end{tabular}

* Countries not included in the panel data analysis. 
Table 2. Ratios of annual values of stock index futures contracts to GDP

\begin{tabular}{|c|c|c|c|c|c|c|c|c|c|c|c|c|}
\hline & 1983 & 1984 & 1985 & 1986 & 1987 & 1988 & 1989 & 1990 & 1991 & 1992 & 1993 & 1994 \\
\hline \multicolumn{13}{|l|}{ Australia } \\
\hline Austria & & & & & & & & & & & 0.001 & 0.001 \\
\hline Belgium & & & & & & & & & & & & 0.000 \\
\hline Brazil & & & & & & & & & & & & 0.090 \\
\hline Canada & & & & & & & & & & 0.002 & 0.002 & 0.004 \\
\hline Denmark & & & & & & & & & & & 0.027 & 0.042 \\
\hline \multicolumn{13}{|l|}{ France } \\
\hline Germany & & & & & & & & & 0.048 & 0.145 & 0.193 & 0.322 \\
\hline Hong Kong, China & & & & & & & 0.053 & 0.052 & 0.124 & 0.251 & 0.680 & 1.873 \\
\hline \multicolumn{13}{|l|}{ Hungary } \\
\hline \multicolumn{13}{|l|}{ Italy } \\
\hline Japan & & & & & & & 0.113 & 0.184 & 0.278 & 0.145 & 0.091 & 0.062 \\
\hline Netherlands & & & & & & & 0.008 & 0.016 & 0.022 & 0.021 & 0.033 & 0.062 \\
\hline \multicolumn{13}{|l|}{ New Zealand } \\
\hline Norway & & & & & & & & & & & 0.001 & 0.000 \\
\hline \multicolumn{13}{|l|}{ Portugal } \\
\hline South Africa & & & & & & & & & 0.007 & 0.015 & 0.021 & 0.062 \\
\hline Spain & & & & & & & & & & & 0.003 & 0.012 \\
\hline Sweden & & & & & & & & & 0.001 & 0.003 & 0.008 & 0.019 \\
\hline Switzerland & & & & & & & & & 0.021 & 0.044 & 0.054 & 0.116 \\
\hline United Kingdom & & & 0.003 & 0.005 & 0.018 & 0.018 & 0.036 & 0.058 & 0.066 & 0.107 & 0.128 & 0.192 \\
\hline United States & 0.021 & 0.037 & 0.049 & 0.063 & 0.077 & 0.040 & 0.038 & 0.043 & 0.047 & 0.051 & 0.054 & 0.067 \\
\hline
\end{tabular}

Table 2. Ratios of annual values of stock index futures contracts to GDP (continued)

\begin{tabular}{lccccccccccc}
\hline & $\mathbf{1 9 9 5}$ & $\mathbf{1 9 9 6}$ & $\mathbf{1 9 9 7}$ & $\mathbf{1 9 9 8}$ & $\mathbf{1 9 9 9}$ & $\mathbf{2 0 0 0}$ & $\mathbf{2 0 0 1}$ & $\mathbf{2 0 0 2}$ & $\mathbf{2 0 0 3}$ & $\mathbf{2 0 0 4}$ & Avg. \\
\hline Australia & & & & & & & 0.085 & 0.096 & 0.095 & 0.110 & 0.097 \\
\hline Austria & 0.002 & 0.002 & 0.003 & 0.003 & 0.003 & 0.002 & 0.001 & 0.001 & 0.000 & 0.000 & 0.002 \\
\hline Belgium & 0.000 & 0.001 & 0.001 & 0.002 & 0.003 & 0.002 & 0.002 & 0.001 & 0.001 & 0.001 & 0.001 \\
\hline Brazil & 0.096 & 0.113 & 0.188 & 0.111 & 0.062 & 0.094 & 0.065 & 0.047 & 0.054 & 0.098 & 0.092 \\
\hline Canada & 0.004 & 0.005 & 0.013 & 0.019 & 0.023 & & & & & & 0.009 \\
\hline Denmark & 0.020 & 0.018 & 0.032 & 0.052 & 0.227 & 0.214 & 0.083 & 0.071 & 0.083 & 0.094 & 0.080 \\
\hline France & & & & & & 0.124 & 0.109 & 0.099 & 0.083 & 0.081 & 0.099 \\
\hline Germany & 0.265 & 0.360 & 0.631 & 0.857 & 0.377 & 0.519 & 0.460 & 0.460 & 0.455 & 0.663 & 0.411 \\
\hline Hong Kong, China & 1.829 & 2.029 & 2.911 & 2.780 & 2.582 & 2.468 & 2.070 & 1.938 & 2.456 & 4.094 & 1.762 \\
\hline Hungary & & 0.004 & 0.058 & 0.159 & 0.094 & 0.079 & 0.023 & 0.013 & 0.014 & 0.021 & 0.052 \\
\hline Italy & 0.000 & 0.000 & 0.000 & 0.000 & 0.000 & 0.000 & 0.000 & 0.000 & 0.000 & 0.000 & 0.000 \\
\hline Japan & 0.064 & 0.073 & 0.059 & 0.042 & 0.063 & 0.056 & 0.047 & 0.046 & 0.048 & 0.069 & 0.090 \\
\hline Netherlands & 0.054 & 0.094 & 0.220 & 0.414 & 0.456 & 0.429 & 0.342 & 0.312 & 0.257 & 0.325 & 0.192 \\
\hline New Zealand & & 0.002 & 0.001 & 0.001 & 0.000 & 0.000 & 0.000 & 0.000 & 0.000 & 0.000 & 0.000 \\
\hline Norway & 0.000 & 0.001 & 0.005 & 0.016 & 0.026 & 0.038 & 0.029 & 0.030 & 0.022 & 0.031 & 0.017 \\
\hline Portugal & & & 0.030 & 0.107 & 0.053 & 0.039 & 0.021 & 0.010 & 0.005 & 0.004 & 0.034 \\
\hline South Africa & 0.050 & 0.043 & 0.055 & 0.084 & 0.107 & 0.113 & 0.137 & 0.179 & 0.128 & 0.174 & 0.084 \\
\hline Spain & 0.011 & 0.014 & 0.008 & 0.008 & 0.006 & 0.005 & 0.003 & 0.002 & 0.002 & 0.002 & 0.006 \\
\hline Sweden & 0.029 & 0.027 & 0.054 & 0.170 & 0.366 & 0.682 & 0.502 & 0.310 & 0.271 & 0.411 & 0.204 \\
\hline Switzerland & 0.110 & 0.166 & 0.232 & 0.384 & 1.266 & 0.916 & 0.778 & 0.878 & 0.934 & 0.999 & 0.493 \\
\hline United Kingdom & 0.161 & 0.170 & 0.223 & 0.364 & 0.593 & 0.679 & 0.639 & 0.704 & 0.723 & 0.794 & 0.284 \\
\hline United States & 0.085 & 0.105 & 0.126 & 0.234 & 0.245 & 0.218 & 0.165 & 0.143 & 0.114 & 0.097 & 0.096 \\
\hline
\end{tabular}


Table 3. Summary statistics (1994-2003)

\begin{tabular}{llll}
\hline & Economic Growth & Futures Markets & Bank Credit \\
\hline Descriptive Statistics & & & \\
Mean & 2.50 & 32.00 & 91.51 \\
Median & 2.63 & 8.78 & 92.21 \\
Maximum & 10.17 & 291.00 & 178.49 \\
Minimum & -5.45 & 0.02 & 26.77 \\
Std. Dev. & 1.84 & 58.62 & 41.24 \\
& & & \\
Observations & 150 & 150 & \\
& & & \\
Correlations & & & \\
Economic Growth & 1.00 & & \\
Futures Markets & 0.04 & 1.00 & 1.00 \\
Bank Credit & 0.24 & 0.57 & \\
\hline
\end{tabular}


Dependent Variable: GDP

Method: Panel Generalize $\bar{d}$ Method of Moments

Transformation: First Differences

Cross-sections included: 15

Instrument list: GDP (-2) FUTURES (-1) BAN (-1) INF (-1) FDI (-1) CRIS (-1)

\begin{tabular}{|c|c|c|c|c|}
\hline Variable & Coefficient & Std. Error & t-Statistic & Prob. \\
\hline GDP_(-1) & 0.781713 & 0.049413 & 15.82002 & 0.0000 \\
\hline FUTURES & 0.030307 & 0.010573 & 2.866468 & 0.0051 \\
\hline $\mathrm{BAN}_{-}$ & 0.023094 & 0.021699 & 1.064257 & 0.2898 \\
\hline $\mathrm{INF}_{-}$ & -0.000681 & 0.000375 & -1.818339 & 0.0720 \\
\hline $\mathrm{FDI}^{-}$ & 0.001374 & 0.000424 & 3.239113 & 0.0016 \\
\hline CRIS & -0.042292 & 0.006941 & -6.093078 & 0.0000 \\
\hline J-statistic & 11.98116 & & & \\
\hline Instrument rank & 15.00000 & & & \\
\hline Sargan test ${ }^{\mathrm{a}}(\mathrm{p}$-val) & 0.214 & & & \\
\hline Serial Correlation test ${ }^{\mathrm{b}}$ ( $\mathrm{p}$-value) & 0.729 & & & \\
\hline
\end{tabular}

GDP : : Logarithm of Real Gross Domestic Product.

FUTURES : Value of futures contracts divided by GDP.

BAN : Value of deposit money bank credits to the private sector divided by GDP.

INF : Inflation.

FDI : Foreign direct investment divided by GDP.

CRIS : Crisis periods dummy.

a Sargan test has the null hypothesis that the over-identifying restrictions are valid.

${ }^{\mathrm{b}}$ Serial correlation test has the null hypothesis that errors in the first-difference regression exhibit no second order serial correlation. 
Table 5. Unit root tests

\begin{tabular}{|c|c|c|c|c|c|c|c|}
\hline \multirow{2}{*}{ Country } & & \multicolumn{3}{|c|}{ FUTURES } & \multicolumn{3}{|c|}{ GDP } \\
\hline & & Statistic & $\begin{array}{c}\text { Critical } \\
\text { Value }(5 \%)\end{array}$ & Lag & Statistic & $\begin{array}{c}\text { Critical } \\
\text { Value }(5 \%)\end{array}$ & Lag \\
\hline \multirow[t]{6}{*}{ Australia } & Level & & & & & & \\
\hline & ADF & 1.92 & -3.83 & 7 & -2.42 & -3.73 & 3 \\
\hline & PP & -2.16 & -3.66 & 7 & -2.76 & -3.67 & 1 \\
\hline & First Diff. ( $\Delta)$ & & & & & & \\
\hline & $\mathrm{ADF}$ & 0.01 & -3.79 & 5 & -1.76 & -3.93 & 7 \\
\hline & PP & -3.39 & -3.67 & 0 & -6.43 & -3.69 & 5 \\
\hline \multirow[t]{6}{*}{ Austria } & Level & & & & & & \\
\hline & ADF & -1.76 & -3.52 & 7 & -1.22 & -3.51 & 1 \\
\hline & PP & -2.02 & -3.50 & 4 & -1.09 & -3.50 & 4 \\
\hline & First Diff. $(\Delta)$ & & & & & & \\
\hline & ADF & -1.77 & -3.52 & 6 & -8.17 & -3.51 & 0 \\
\hline & PP & -7.66 & -3.50 & 4 & -8.74 & -3.51 & 7 \\
\hline \multirow[t]{6}{*}{ Belgium } & Level & & & & & & \\
\hline & ADF & -3.39 & -3.53 & 7 & -1.44 & -3.51 & 0 \\
\hline & PP & -1.40 & -3.51 & 3 & -1.47 & -3.51 & 2 \\
\hline & First Diff. $(\Delta)$ & & & & & & \\
\hline & ADF & -1.67 & -3.52 & 3 & -7.11 & -3.52 & 0 \\
\hline & $\mathrm{PP}$ & -7.53 & -3.51 & 3 & -7.11 & -3.52 & 1 \\
\hline \multirow[t]{6}{*}{ Brazil } & Level & & & & & & \\
\hline & $\mathrm{ADF}$ & -1.98 & -3.53 & 8 & -1.87 & -3.50 & 3 \\
\hline & PP & -2.84 & -3.51 & 1 & -7.51 & -3.49 & 12 \\
\hline & First Diff. $(\Delta)$ & & & & & & \\
\hline & $\mathrm{ADF}$ & -3.45 & -3.53 & 7 & -16.38 & -3.50 & 2 \\
\hline & PP & -7.41 & -3.51 & 7 & -23.82 & -3.50 & 12 \\
\hline \multirow[t]{6}{*}{ Canada } & Level & & & & & & \\
\hline & ADF & -0.28 & -3.61 & 10 & -1.55 & -3.55 & 1 \\
\hline & PP & -1.80 & -3.55 & 1 & -1.07 & -3.54 & 3 \\
\hline & First Diff. $(\Delta)$ & & & & & & \\
\hline & $\mathrm{ADF}$ & -2.96 & -3.61 & 9 & -3.14 & -3.55 & 0 \\
\hline & $\mathrm{PP}$ & -2.98 & -3.55 & 1 & -3.16 & -3.55 & 2 \\
\hline \multirow[t]{6}{*}{ Denmark } & Level & & & & & & \\
\hline & ADF & -2.55 & -3.50 & 0 & -1.09 & -3.51 & 3 \\
\hline & PP & -2.61 & -3.50 & 4 & -1.68 & -3.50 & 4 \\
\hline & First Diff. $(\Delta)$ & & & & & & \\
\hline & $\mathrm{ADF}$ & -6.57 & -3.50 & 1 & -6.41 & -3.51 & 2 \\
\hline & PP & -8.72 & -3.50 & 3 & -18.65 & -3.50 & 23 \\
\hline \multirow[t]{6}{*}{ France } & Level & & & & & & \\
\hline & $\mathrm{ADF}$ & -1.03 & -3.71 & 8 & -0.21 & -3.79 & 9 \\
\hline & PP & -2.37 & -3.60 & 1 & -10.35 & -3.62 & 12 \\
\hline & First Diff. $(\Delta)$ & & & & & & \\
\hline & $\mathrm{ADF}$ & -1.00 & -3.76 & 9 & -0.45 & -3.83 & 9 \\
\hline & PP & -4.49 & -3.61 & 0 & -20.57 & -3.63 & 11 \\
\hline \multirow[t]{6}{*}{ Germany } & Level & & & & & & \\
\hline & ADF & -2.23 & -3.50 & 6 & -2.10 & -3.49 & 0 \\
\hline & PP & -3.22 & -3.49 & 3 & -2.23 & -3.49 & 1 \\
\hline & First Diff. $(\Delta)$ & & & & & & \\
\hline & $\mathrm{ADF}$ & -4.69 & -3.50 & 4 & -6.93 & -3.49 & 0 \\
\hline & PP & -11.19 & -3.49 & 11 & -6.93 & -3.49 & 0 \\
\hline
\end{tabular}


Table 5. Unit root tests (continued)

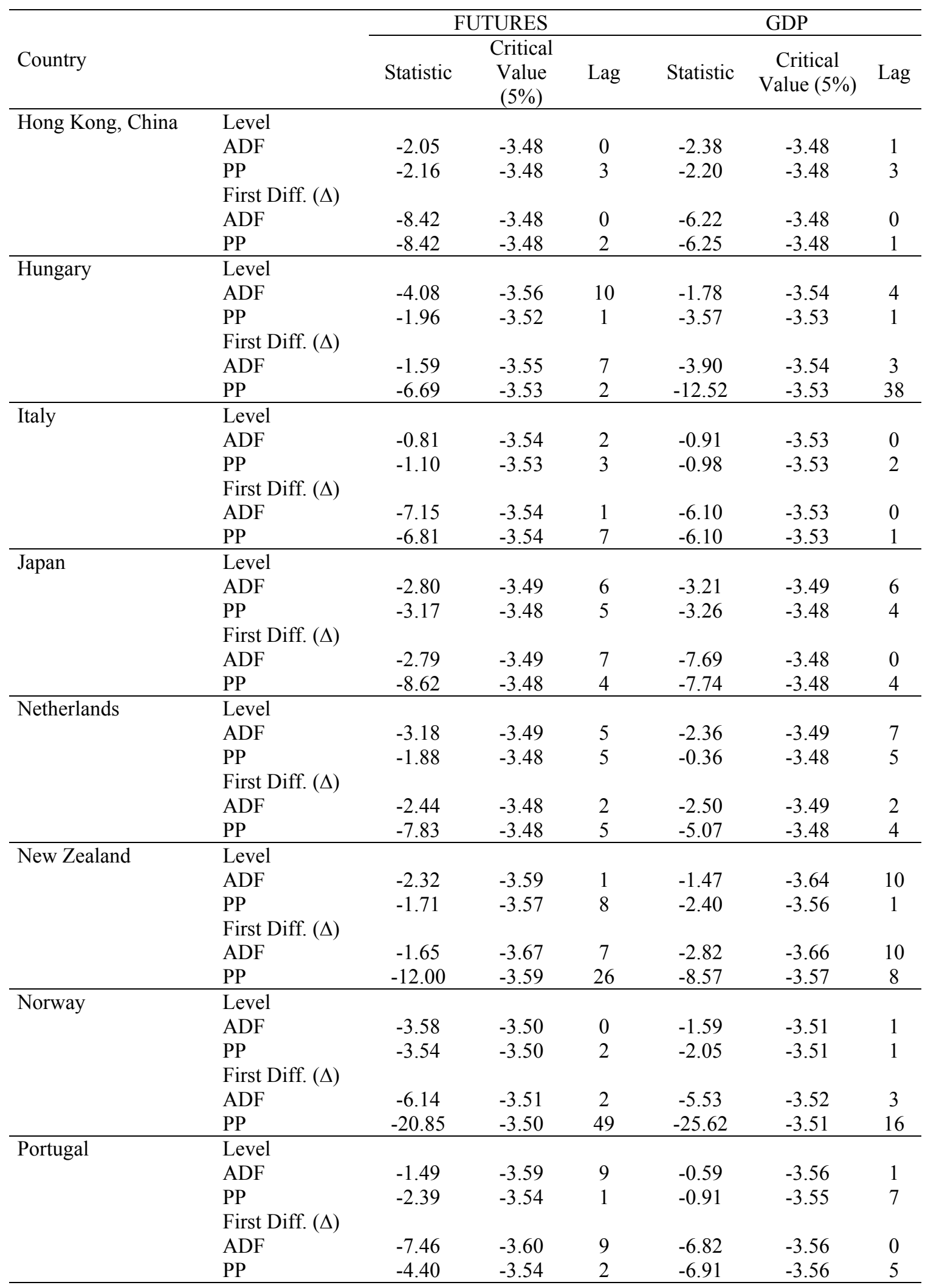


Table 5. Unit root tests (continued)

\begin{tabular}{|c|c|c|c|c|c|c|c|}
\hline \multirow[b]{2}{*}{ Country } & & \multicolumn{3}{|c|}{ FUTURES } & \multicolumn{3}{|c|}{ GDP } \\
\hline & & Statistic & $\begin{array}{c}\text { Critical } \\
\text { Value }(5 \%)\end{array}$ & Lag & Statistic & $\begin{array}{c}\text { Critical } \\
\text { Value }(5 \%)\end{array}$ & Lag \\
\hline \multirow[t]{6}{*}{ South Africa } & Level & & & & & & \\
\hline & $\mathrm{ADF}$ & -4.90 & -3.49 & 0 & -3.85 & -3.49 & 1 \\
\hline & $\mathrm{PP}$ & -4.92 & -3.49 & 1 & -3.03 & -3.49 & 2 \\
\hline & First Diff. $(\Delta)$ & & & & & & \\
\hline & $\mathrm{ADF}$ & -5.20 & -3.50 & 6 & -3.82 & -3.50 & 2 \\
\hline & $\mathrm{PP}$ & -17.45 & -3.49 & 17 & -3.36 & -3.49 & 5 \\
\hline \multirow[t]{6}{*}{ Spain } & Level & & & & & & \\
\hline & $\mathrm{ADF}$ & -3.20 & -3.50 & 0 & -2.08 & -3.51 & 4 \\
\hline & $\mathrm{PP}$ & -3.14 & -3.50 & 1 & -8.45 & -3.50 & 0 \\
\hline & First Diff. $(\Delta)$ & & & & & & \\
\hline & $\mathrm{ADF}$ & -3.97 & -3.51 & 4 & -4.55 & -3.51 & 3 \\
\hline & $\mathrm{PP}$ & -8.52 & -3.50 & 0 & -26.07 & -3.50 & 15 \\
\hline \multirow[t]{6}{*}{ Sweden } & Level & & & & & & \\
\hline & $\mathrm{ADF}$ & -1.80 & -3.49 & 3 & -3.26 & -3.49 & 4 \\
\hline & $\mathrm{PP}$ & -1.86 & -3.49 & 5 & -2.84 & -3.49 & 9 \\
\hline & First Diff. $(\Delta)$ & & & & & & \\
\hline & $\mathrm{ADF}$ & -2.18 & -3.49 & 2 & -2.49 & -3.49 & 3 \\
\hline & $\mathrm{PP}$ & -9.70 & -3.49 & 5 & -9.41 & -3.49 & 7 \\
\hline \multirow[t]{6}{*}{ Switzerland } & Level & & & & & & \\
\hline & $\mathrm{ADF}$ & -2.61 & -3.49 & 0 & -2.08 & -3.49 & 1 \\
\hline & $\mathrm{PP}$ & -2.69 & -3.49 & 1 & -2.40 & -3.49 & 2 \\
\hline & First Diff. $(\Delta)$ & & & & & & \\
\hline & $\mathrm{ADF}$ & -7.52 & -3.49 & 0 & -5.64 & -3.49 & 0 \\
\hline & $\mathrm{PP}$ & -7.54 & -3.49 & 3 & -5.69 & -3.49 & 1 \\
\hline \multirow[t]{6}{*}{ United Kingdom } & Level & & & & & & \\
\hline & $\mathrm{ADF}$ & -1.62 & -3.47 & 9 & -1.65 & -3.47 & 7 \\
\hline & $\mathrm{PP}$ & -2.92 & -3.46 & 7 & -6.42 & -3.47 & 9 \\
\hline & First Diff. $(\Delta)$ & & & & & & \\
\hline & $\mathrm{ADF}$ & -3.63 & -3.47 & 8 & -2.51 & -3.47 & 6 \\
\hline & $\mathrm{PP}$ & -17.16 & -3.46 & 14 & -12.85 & -3.47 & 13 \\
\hline \multirow[t]{6}{*}{ United States } & Level & & & & & & \\
\hline & $\mathrm{ADF}$ & -1.23 & -3.46 & 0 & -3.23 & -3.47 & 9 \\
\hline & $\mathrm{PP}$ & -1.24 & -3.46 & 2 & -2.81 & -3.46 & 5 \\
\hline & First Diff. $(\Delta)$ & & & & & & \\
\hline & $\mathrm{ADF}$ & -9.55 & -3.46 & 0 & -3.33 & -3.47 & 10 \\
\hline & $\mathrm{PP}$ & -9.55 & -3.46 & 1 & -6.65 & -3.46 & 3 \\
\hline
\end{tabular}

Data : Quarterly data are used.

FUTURES : Value of futures contracts divided by GDP.

GDP : Logarithm of Real Gross Domestic Product (seasonalized).

ADF : Augmented Dickey-Fuller Test $\left(\mathrm{H}_{0}\right.$ : There is unit root).

PP : Phillips-Perron Test $\left(\mathrm{H}_{0}\right.$ : There is unit root).

First Diff. $(\Delta) \quad$ : First difference operator or the change in the variable.

If test statistic $>$ critical value, then $\mathrm{H}_{0}$ is rejected. 
Table 6. Johansen cointegration tests

\begin{tabular}{|c|c|c|}
\hline Country & Hypothesized Number of CE(s) & Probability \\
\hline \multirow[t]{2}{*}{ Australia } & None & $0.04 * *$ \\
\hline & At most 1 & 0.22 \\
\hline \multirow[t]{2}{*}{ Austria } & None & $0.01 * * *$ \\
\hline & At most 1 & $0.02 * *$ \\
\hline \multirow[t]{2}{*}{ Belgium } & None & $0.01 * * *$ \\
\hline & At most 1 & $0.02 * *$ \\
\hline \multirow[t]{2}{*}{ Brazil } & None & $0.05 * *$ \\
\hline & At most 1 & 0.99 \\
\hline \multirow[t]{2}{*}{ Canada } & None & $0.00 * * *$ \\
\hline & At most 1 & 0.47 \\
\hline \multirow[t]{2}{*}{ Denmark } & None & $0.00 * * *$ \\
\hline & At most 1 & $0.01 * * *$ \\
\hline \multirow[t]{2}{*}{ France } & None & $0.00 * * *$ \\
\hline & At most 1 & 0.04 \\
\hline \multirow[t]{2}{*}{ Germany } & None & $0.00 * * *$ \\
\hline & At most 1 & 0.13 \\
\hline \multirow[t]{2}{*}{ Hong Kong, China } & None & $0.02 * *$ \\
\hline & At most 1 & 0.18 \\
\hline \multirow[t]{2}{*}{ Hungary } & None & $0.00 * * *$ \\
\hline & At most 1 & 0.37 \\
\hline \multirow[t]{2}{*}{ Italy } & None & $0.00 * * *$ \\
\hline & At most 1 & 0.73 \\
\hline \multirow[t]{2}{*}{ Japan } & None & $0.01 * * *$ \\
\hline & At most 1 & 0.41 \\
\hline \multirow[t]{2}{*}{ Netherlands } & None & $0.03 * *$ \\
\hline & At most 1 & 0.20 \\
\hline \multirow[t]{2}{*}{ New Zealand } & None & $0.00 * * *$ \\
\hline & At most 1 & 0.37 \\
\hline \multirow[t]{2}{*}{ Norway } & None & $0.04 * *$ \\
\hline & At most 1 & $0.01 * * *$ \\
\hline \multirow[t]{2}{*}{ Portugal } & None & $0.00 * * *$ \\
\hline & At most 1 & $0.00 * * *$ \\
\hline \multirow[t]{2}{*}{ South Africa } & None & $0.03 * *$ \\
\hline & At most 1 & 0.35 \\
\hline \multirow[t]{2}{*}{ Spain } & None & $0.01 * * *$ \\
\hline & At most 1 & 0.80 \\
\hline \multirow[t]{2}{*}{ Sweden } & None & $0.09 *$ \\
\hline & At most 1 & 0.45 \\
\hline \multirow[t]{2}{*}{ Switzerland } & None & $0.02 * *$ \\
\hline & At most 1 & 0.49 \\
\hline \multirow[t]{2}{*}{ United Kingdom } & None & $0.05 * *$ \\
\hline & At most 1 & 0.68 \\
\hline \multirow[t]{2}{*}{ United States } & None & $0.04 * *$ \\
\hline & At most 1 & 0.61 \\
\hline
\end{tabular}

Series : FUTURES (value of futures contracts divided by GDP), GDP (logarithm of seasonalized real gross domestic product).

Data : Quarterly Level data.

CE : Cointegrating Equation.

$*, * *$, and $* * *$ stand for the significance at $10 \%, 5 \%$, and $1 \%$ level, respectively. 
Table 7. Granger-causality tests

\begin{tabular}{|c|c|c|c|}
\hline Country & Variable and Direction & Probability & Lag \\
\hline \multirow[t]{2}{*}{ Australia } & $\Delta$ FUTURES $\Rightarrow \Delta$ GDP & 0.60 & 4 \\
\hline & $\Delta \mathrm{GDP} \Rightarrow \Delta \mathrm{FUTURES}$ & 0.76 & 4 \\
\hline \multirow[t]{2}{*}{ Austria } & $\Delta$ FUTURES $\Rightarrow \Delta$ GDP & 0.49 & 8 \\
\hline & $\Delta \mathrm{GDP} \Rightarrow \Delta \mathrm{FUTURES}$ & 0.29 & 8 \\
\hline \multirow[t]{2}{*}{ Belgium } & $\Delta$ FUTURES $\Rightarrow \Delta$ GDP & 0.11 & 10 \\
\hline & $\Delta \mathrm{GDP} \Rightarrow \Delta \mathrm{FUTURES}$ & 0.33 & 10 \\
\hline \multirow[t]{2}{*}{ Brazil } & $\Delta$ FUTURES $\Rightarrow \Delta$ GDP & $0.02 * *$ & 8 \\
\hline & $\Delta \mathrm{GDP} \Rightarrow \Delta \mathrm{FUTURES}$ & 0.39 & 8 \\
\hline \multirow[t]{2}{*}{ Canada } & $\Delta$ FUTURES $\Rightarrow \Delta$ GDP & 0.71 & 9 \\
\hline & $\Delta \mathrm{GDP} \Rightarrow \Delta \mathrm{FUTURES}$ & 0.76 & 9 \\
\hline \multirow[t]{2}{*}{ Denmark } & $\Delta$ FUTURES $\Rightarrow \Delta$ GDP & 0.33 & 7 \\
\hline & $\Delta \mathrm{GDP} \Rightarrow \Delta \mathrm{FUTURES}$ & 0.83 & 7 \\
\hline \multirow[t]{2}{*}{ France } & $\Delta$ FUTURES $\Rightarrow \Delta$ GDP & $0.02 * *$ & 3 \\
\hline & $\Delta \mathrm{GDP} \Rightarrow \Delta \mathrm{FUTURES}$ & 0.16 & 3 \\
\hline \multirow[t]{2}{*}{ Germany } & $\Delta$ FUTURES $\Rightarrow \Delta$ GDP & 0.55 & 9 \\
\hline & $\Delta \mathrm{GDP} \Rightarrow \Delta \mathrm{FUTURES}$ & 0.47 & 9 \\
\hline \multirow[t]{2}{*}{ Hong Kong, China } & $\Delta$ FUTURES $\Rightarrow \Delta$ GDP & 0.82 & 1 \\
\hline & $\Delta \mathrm{GDP} \Rightarrow \Delta \mathrm{FUTURES}$ & $0.02 * *$ & 1 \\
\hline \multirow[t]{2}{*}{ Hungary } & $\Delta$ FUTURES $\Rightarrow \Delta$ GDP & 0.52 & 10 \\
\hline & $\Delta \mathrm{GDP} \Rightarrow \Delta \mathrm{FUTURES}$ & 0.28 & 10 \\
\hline \multirow[t]{2}{*}{ Italy } & $\Delta$ FUTURES $\Rightarrow \Delta$ GDP & 0.70 & 10 \\
\hline & $\Delta \mathrm{GDP} \Rightarrow \Delta \mathrm{FUTURES}$ & 0.12 & 10 \\
\hline \multirow[t]{2}{*}{ Japan } & $\Delta$ FUTURES $\Rightarrow \Delta$ GDP & $0.05 * *$ & 8 \\
\hline & $\Delta \mathrm{GDP} \Rightarrow \Delta \mathrm{FUTURES}$ & 0.67 & 8 \\
\hline \multirow[t]{2}{*}{ Netherlands } & $\Delta \mathrm{FUTURES} \Rightarrow \Delta \mathrm{GDP}$ & 0.52 & 1 \\
\hline & $\Delta \mathrm{GDP} \Rightarrow \Delta \mathrm{FUTURES}$ & $0.08 *$ & 1 \\
\hline \multirow[t]{2}{*}{ New Zealand } & $\Delta$ FUTURES $\Rightarrow \Delta$ GDP & 0.49 & 4 \\
\hline & $\Delta \mathrm{GDP} \Rightarrow \Delta \mathrm{FUTURES}$ & 0.59 & 4 \\
\hline \multirow[t]{2}{*}{ Norway } & $\Delta$ FUTURES $\Rightarrow \Delta$ GDP & 0.64 & 1 \\
\hline & $\Delta \mathrm{GDP} \Rightarrow \Delta \mathrm{FUTURES}$ & $0.02 * *$ & 1 \\
\hline \multirow[t]{2}{*}{ Portugal } & $\Delta$ FUTURES $\Rightarrow \Delta$ GDP & $0.03 * *$ & 8 \\
\hline & $\Delta \mathrm{GDP} \Rightarrow \Delta \mathrm{FUTURES}$ & $0.02 * *$ & 8 \\
\hline \multirow[t]{2}{*}{ South Africa } & $\Delta$ FUTURES $\Rightarrow \Delta$ GDP & 0.60 & 1 \\
\hline & $\Delta \mathrm{GDP} \Rightarrow \Delta \mathrm{FUTURES}$ & 0.77 & 1 \\
\hline \multirow[t]{2}{*}{ Spain } & $\Delta$ FUTURES $\Rightarrow \Delta$ GDP & 0.39 & 2 \\
\hline & $\Delta \mathrm{GDP} \Rightarrow \Delta \mathrm{FUTURES}$ & 0.88 & 2 \\
\hline \multirow[t]{2}{*}{ Sweden } & $\Delta$ FUTURES $\Rightarrow \Delta$ GDP & 0.57 & 3 \\
\hline & $\Delta \mathrm{GDP} \Rightarrow \Delta \mathrm{FUTURES}$ & 0.59 & 3 \\
\hline \multirow[t]{2}{*}{ Switzerland } & $\Delta$ FUTURES $\Rightarrow \Delta$ GDP & 0.95 & 1 \\
\hline & $\Delta \mathrm{GDP} \Rightarrow \Delta \mathrm{FUTURES}$ & 0.12 & 1 \\
\hline \multirow[t]{2}{*}{ United Kingdom } & $\Delta \mathrm{FUTURES} \Rightarrow \Delta \mathrm{GDP}$ & 0.34 & 9 \\
\hline & $\Delta \mathrm{GDP} \Rightarrow \Delta \mathrm{FUTURES}$ & 0.16 & 9 \\
\hline \multirow[t]{2}{*}{ United States } & $\Delta \mathrm{FUTURES} \Rightarrow \Delta \mathrm{GDP}$ & 0.60 & 3 \\
\hline & $\Delta \mathrm{GDP} \Rightarrow \Delta \mathrm{FUTURES}$ & 0.62 & 3 \\
\hline
\end{tabular}

Data $\quad$ : First differenced quarterly data.

Lags $\quad: 1-10$ lags are tried, the lag minimizing the Akaike Information Criterion (AIC) is chosen. First Diff. $(\Delta)$ : First difference operator or the change in the variable.

Null hypothesis: i) change in FUTURES does not Granger-cause change in GDP, ii) change in GDP does not Granger-cause change in FUTURES.

$*, * *$, and $* * *$ stand for the significance at $10 \%, 5 \%$, and $1 \%$ level, respectively. 
Table 8. VECM regression results

\begin{tabular}{|c|c|c|c|c|}
\hline \multirow[t]{2}{*}{ Country } & \multicolumn{2}{|c|}{ Cointegration Equation } & \multicolumn{2}{|c|}{ Adjustment Parameter } \\
\hline & & Coefficient Estimate & & Coefficient Estimate \\
\hline \multirow[t]{3}{*}{ Australia } & FUT $(-1)$ & 1.000 & $\Delta \mathrm{FUT}$ & 0.362 \\
\hline & $\operatorname{GDP}(-1)$ & 0.254 & $\Delta \mathrm{GDP}$ & -0.202 \\
\hline & Constant & -7.041 & & \\
\hline \multirow[t]{3}{*}{ Austria } & FUT $(-1)$ & 1.000 & $\Delta$ FUT & -0.024 \\
\hline & $\operatorname{GDP}(-1)$ & 0.129 & $\Delta \mathrm{GDP}$ & -0.780 \\
\hline & Constant & -3.532 & & \\
\hline \multirow[t]{3}{*}{ Belgium } & FUT (-1) & 1.000 & $\Delta$ FUT & -0.054 \\
\hline & $\operatorname{GDP}(-1)$ & -0.004 & $\Delta \mathrm{GDP}$ & 4.678 \\
\hline & Constant & 0.110 & & \\
\hline \multirow[t]{3}{*}{ Brazil } & FUT (-1) & 1.000 & $\Delta \mathrm{FUT}$ & -0.716 \\
\hline & $\operatorname{GDP}(-1)$ & 0.642 & $\Delta \mathrm{GDP}$ & 0.460 \\
\hline & Constant & -16.726 & & \\
\hline \multirow[t]{3}{*}{ Canada } & FUT (-1) & 1.000 & $\Delta$ FUT & -1.093 \\
\hline & $\operatorname{GDP}(-1)$ & -0.074 & $\Delta \mathrm{GDP}$ & 1.801 \\
\hline & Constant & 2.014 & & \\
\hline \multirow[t]{3}{*}{ Denmark } & FUT (-1) & 1.000 & $\Delta \mathrm{FUT}$ & -0.022 \\
\hline & $\operatorname{GDP}(-1)$ & 12.691 & $\Delta \mathrm{GDP}$ & -0.008 \\
\hline & Constant & -335.796 & & \\
\hline \multirow[t]{3}{*}{ France } & FUT (-1) & 1.000 & $\Delta$ FUT & -0.282 \\
\hline & $\operatorname{GDP}(-1)$ & 0.729 & $\Delta \mathrm{GDP}$ & -0.644 \\
\hline & Constant & -20.902 & & \\
\hline \multirow[t]{3}{*}{ Germany } & FUT (-1) & 1.000 & $\Delta \mathrm{FUT}$ & -0.427 \\
\hline & $\operatorname{GDP}(-1)$ & -3.199 & $\Delta \mathrm{GDP}$ & 0.032 \\
\hline & Constant & 87.824 & & \\
\hline \multirow[t]{3}{*}{ Hong Kong, China } & FUT (-1) & 1.000 & $\Delta$ FUT & -0.166 \\
\hline & $\operatorname{GDP}(-1)$ & -6.788 & $\Delta \mathrm{GDP}$ & -0.002 \\
\hline & Constant & 177.167 & & \\
\hline \multirow[t]{3}{*}{ Hungary } & FUT (-1) & 1.000 & $\Delta$ FUT & -1.950 \\
\hline & $\operatorname{GDP}(-1)$ & 0.298 & $\Delta \mathrm{GDP}$ & 0.263 \\
\hline & Constant & -8.642 & & \\
\hline \multirow[t]{3}{*}{ Italy } & FUT (-1) & 1.000 & $\Delta \mathrm{FUT}$ & -0.347 \\
\hline & $\operatorname{GDP}(-1)$ & 0.004 & $\Delta \mathrm{GDP}$ & -6.228 \\
\hline & Constant & -0.128 & & \\
\hline \multirow[t]{3}{*}{ Japan } & FUT (-1) & 1.000 & $\Delta$ FUT & -0.197 \\
\hline & $\operatorname{GDP}(-1)$ & 0.178 & $\Delta \mathrm{GDP}$ & 0.026 \\
\hline & Constant & -6.090 & & \\
\hline \multirow[t]{3}{*}{ Netherlands } & FUT (-1) & 1.000 & $\Delta$ FUT & -0.106 \\
\hline & $\operatorname{GDP}(-1)$ & -1.612 & $\Delta \mathrm{GDP}$ & 0.020 \\
\hline & Constant & 41.705 & & \\
\hline \multirow[t]{3}{*}{ New Zealand } & FUT (-1) & 1.000 & $\Delta$ FUT & -1.270 \\
\hline & $\operatorname{GDP}(-1)$ & 0.003 & $\Delta \mathrm{GDP}$ & -54.787 \\
\hline & Constant & -0.069 & & \\
\hline \multirow[t]{3}{*}{ Norway } & FUT (-1) & 1.000 & $\Delta \mathrm{FUT}$ & -0.418 \\
\hline & $\operatorname{GDP}(-1)$ & -0.104 & $\Delta \mathrm{GDP}$ & -0.295 \\
\hline & Constant & 2.735 & & \\
\hline \multirow[t]{3}{*}{ Portugal } & FUT (-1) & 1.000 & $\Delta$ FUT & -0.028 \\
\hline & GDP (-1) & 1.615 & $\Delta \mathrm{GDP}$ & -0.212 \\
\hline & Constant & -47.480 & & \\
\hline \multirow[t]{3}{*}{ South Africa } & FUT (-1) & 1.000 & $\Delta$ FUT & -0.529 \\
\hline & $\operatorname{GDP}(-1)$ & -0.454 & $\Delta \mathrm{GDP}$ & 0.042 \\
\hline & Constant & 12.388 & & \\
\hline
\end{tabular}


Table 8. VECM regression (continued)

\begin{tabular}{lllll}
\hline Country & \multicolumn{2}{l}{ Cointegration Equation } & \multicolumn{2}{l}{ Adjustment Parameter } \\
\hline Spain & & Coefficient Estimate & & Coefficient Estimate \\
& FUT (-1) & 1.000 & $\Delta$ FUT & -0.507 \\
& GDP (-1) & 0.032 & $\Delta$ GDP & 0.818 \\
& Constant & -0.994 & & \\
\hline Sweden & FUT (-1) & 1.000 & $\Delta$ FUT & -0.242 \\
& GDP (-1) & -1.407 & $\Delta$ GDP & -0.003 \\
& Constant & 37.693 & & \\
\hline Switzerland & FUT (-1) & 1.000 & $\Delta$ FUT & -0.226 \\
& GDP (-1) & -6.941 & $\Delta$ GDP & 0.010 \\
& Constant & 175.208 & & \\
\hline United Kingdom & FUT (-1) & 1.000 & $\Delta$ FUT & -0.226 \\
& GDP (-1) & -2.246 & $\Delta$ GDP & 0.000 \\
& Constant & 58.245 & & \\
\hline United States & FUT (-1) & 1.000 & $\Delta$ FUT & -0.063 \\
& GDP (-1) & -0.146 & $\Delta$ GDP & -0.003
\end{tabular}

VECM is constructed by adding an adjustment parameter to the cointegration equation, consequently short-run adjustments correct deviations from the long-run equilibrium. Two important results of the VECM regression are the coefficients of cointegration equations and adjustment parameters.

First Diff. $(\Delta) \quad$ : First difference operator or the change in the variable. 
Table 9. Variance decomposition

\begin{tabular}{|c|c|c|c|c|c|c|c|}
\hline \multirow[t]{2}{*}{ Country } & \multirow[t]{2}{*}{ Period } & \multicolumn{3}{|c|}{$\begin{array}{l}\text { Column (a) } \\
\text { FUTURES }\end{array}$} & \multicolumn{3}{|c|}{$\begin{array}{l}\text { Column (b) } \\
\text { GDP }\end{array}$} \\
\hline & & S.E. & $\begin{array}{c}\varepsilon_{1 \mathrm{t}} \\
\text { (FUTURES) }\end{array}$ & $\begin{array}{c}\varepsilon_{2 \mathrm{t}} \\
(\mathrm{GDP})\end{array}$ & S.E. & $\begin{array}{c}\varepsilon_{1 \mathrm{t}} \\
\text { (FUTURES) }\end{array}$ & $\begin{array}{c}\varepsilon_{2 \mathrm{t}} \\
(\mathrm{GDP})\end{array}$ \\
\hline \multirow[t]{10}{*}{ Australia } & 1 & 0.00 & 100.00 & 0.00 & 0.00 & 4.50 & 95.50 \\
\hline & 2 & 0.01 & 98.57 & 1.43 & 0.01 & 3.13 & 96.87 \\
\hline & 3 & 0.01 & 98.85 & 1.15 & 0.01 & 3.49 & 96.51 \\
\hline & 4 & 0.01 & 96.48 & 3.52 & 0.01 & 6.60 & 93.40 \\
\hline & 5 & 0.01 & 91.10 & 8.90 & 0.01 & 7.96 & 92.04 \\
\hline & 6 & 0.02 & 92.20 & 7.80 & 0.01 & 14.65 & 85.35 \\
\hline & 7 & 0.02 & 94.10 & 5.90 & 0.01 & 18.78 & 81.22 \\
\hline & 8 & 0.02 & 95.43 & 4.57 & 0.01 & 19.98 & 80.02 \\
\hline & 9 & 0.03 & 96.43 & 3.57 & 0.01 & 27.33 & 72.67 \\
\hline & 10 & 0.04 & 96.49 & 3.51 & 0.01 & 37.71 & 62.29 \\
\hline \multirow[t]{10}{*}{ Austria } & 1 & 0.00 & 100.00 & 0.00 & 0.01 & 8.06 & 91.94 \\
\hline & 2 & 0.00 & 99.83 & 0.17 & 0.01 & 6.55 & 93.45 \\
\hline & 3 & 0.00 & 96.53 & 3.47 & 0.01 & 5.00 & 95.00 \\
\hline & 4 & 0.00 & 87.59 & 12.41 & 0.01 & 4.84 & 95.16 \\
\hline & 5 & 0.00 & 76.82 & 23.18 & 0.01 & 4.56 & 95.44 \\
\hline & 6 & 0.00 & 70.47 & 29.53 & 0.01 & 5.01 & 94.99 \\
\hline & 7 & 0.00 & 61.21 & 38.79 & 0.02 & 4.95 & 95.05 \\
\hline & 8 & 0.00 & 58.35 & 41.65 & 0.02 & 4.86 & 95.14 \\
\hline & 9 & 0.00 & 60.17 & 39.83 & 0.02 & 4.88 & 95.12 \\
\hline & 10 & 0.00 & 61.00 & 39.00 & 0.02 & 4.93 & 95.07 \\
\hline \multirow[t]{10}{*}{ Belgium } & 1 & 0.00 & 100.00 & 0.00 & 0.01 & 18.74 & 81.26 \\
\hline & 2 & 0.00 & 98.77 & 1.23 & 0.02 & 11.07 & 88.93 \\
\hline & 3 & 0.00 & 98.49 & 1.51 & 0.02 & 10.56 & 89.44 \\
\hline & 4 & 0.00 & 98.36 & 1.64 & 0.02 & 12.16 & 87.84 \\
\hline & 5 & 0.00 & 98.23 & 1.77 & 0.03 & 14.52 & 85.48 \\
\hline & 6 & 0.00 & 98.13 & 1.87 & 0.03 & 17.44 & 82.56 \\
\hline & 7 & 0.00 & 98.04 & 1.96 & 0.03 & 20.66 & 79.34 \\
\hline & 8 & 0.00 & 97.95 & 2.05 & 0.03 & 24.00 & 76.00 \\
\hline & 9 & 0.00 & 97.87 & 2.13 & 0.04 & 27.36 & 72.64 \\
\hline & 10 & 0.00 & 97.79 & 2.21 & 0.04 & 30.65 & 69.35 \\
\hline \multirow[t]{10}{*}{ Brazil } & 1 & 0.02 & 100.00 & 0.00 & 0.02 & 19.12 & 80.88 \\
\hline & 2 & 0.03 & 100.00 & 0.00 & 0.03 & 18.82 & 81.18 \\
\hline & 3 & 0.04 & 92.78 & 7.22 & 0.03 & 26.07 & 73.93 \\
\hline & 4 & 0.04 & 86.06 & 13.94 & 0.03 & 44.91 & 55.09 \\
\hline & 5 & 0.04 & 81.50 & 18.50 & 0.03 & 42.58 & 57.42 \\
\hline & 6 & 0.04 & 80.76 & 19.24 & 0.04 & 47.30 & 52.70 \\
\hline & 7 & 0.04 & 83.04 & 16.96 & 0.04 & 46.96 & 53.04 \\
\hline & 8 & 0.05 & 85.95 & 14.05 & 0.04 & 48.20 & 51.80 \\
\hline & 9 & 0.05 & 85.94 & 14.06 & 0.04 & 41.39 & 58.61 \\
\hline & 10 & 0.05 & 85.28 & 14.72 & 0.05 & 44.70 & 55.30 \\
\hline \multirow[t]{10}{*}{ Canada } & 1 & 0.00 & 100.00 & 0.00 & 0.00 & 3.90 & 96.10 \\
\hline & 2 & 0.00 & 92.57 & 7.43 & 0.01 & 12.89 & 87.11 \\
\hline & 3 & 0.00 & 95.67 & 4.33 & 0.01 & 10.67 & 89.33 \\
\hline & 4 & 0.00 & 97.13 & 2.87 & 0.01 & 13.93 & 86.07 \\
\hline & 5 & 0.00 & 72.67 & 27.33 & 0.01 & 11.90 & 88.10 \\
\hline & 6 & 0.00 & 65.40 & 34.60 & 0.01 & 14.18 & 85.82 \\
\hline & 7 & 0.00 & 47.31 & 52.69 & 0.01 & 14.47 & 85.53 \\
\hline & 8 & 0.00 & 39.89 & 60.11 & 0.01 & 14.91 & 85.09 \\
\hline & 9 & 0.00 & 27.09 & 72.91 & 0.01 & 14.77 & 85.23 \\
\hline & 10 & 0.00 & 24.13 & 75.87 & 0.01 & 13.86 & 86.14 \\
\hline
\end{tabular}


Table 9. Variance decomposition (continued)

\begin{tabular}{|c|c|c|c|c|c|c|c|}
\hline \multirow[t]{2}{*}{ Country } & \multirow[t]{2}{*}{ Period } & \multicolumn{3}{|c|}{$\begin{array}{l}\text { Column (a) } \\
\text { FUTURES }\end{array}$} & \multicolumn{3}{|c|}{$\begin{array}{l}\text { Column (b) } \\
\text { GDP }\end{array}$} \\
\hline & & S.E. & $\begin{array}{c}\varepsilon_{1 \mathrm{t}} \\
\text { (FUTURES) }\end{array}$ & $\begin{array}{c}\varepsilon_{2 t} \\
(\mathrm{GDP})\end{array}$ & S.E. & $\begin{array}{c}\varepsilon_{1 \mathrm{t}} \\
\text { (FUTURES) }\end{array}$ & $\begin{array}{c}\varepsilon_{2 t} \\
(\mathrm{GDP})\end{array}$ \\
\hline \multirow[t]{10}{*}{ Denmark } & 1 & 0.05 & 100.00 & 0.00 & 0.01 & 3.43 & 96.57 \\
\hline & 2 & 0.06 & 97.83 & 2.17 & 0.01 & 4.05 & 95.95 \\
\hline & 3 & 0.07 & 96.70 & 3.30 & 0.01 & 7.45 & 92.55 \\
\hline & 4 & 0.08 & 92.00 & 8.00 & 0.01 & 7.90 & 92.10 \\
\hline & 5 & 0.09 & 91.57 & 8.43 & 0.01 & 8.75 & 91.25 \\
\hline & 6 & 0.10 & 90.86 & 9.14 & 0.01 & 10.34 & 89.66 \\
\hline & 7 & 0.11 & 90.27 & 9.73 & 0.01 & 29.22 & 70.78 \\
\hline & 8 & 0.12 & 90.01 & 9.99 & 0.01 & 38.63 & 61.37 \\
\hline & 9 & 0.13 & 90.16 & 9.84 & 0.01 & 36.86 & 63.14 \\
\hline & 10 & 0.13 & 89.74 & 10.26 & 0.01 & 38.52 & 61.48 \\
\hline \multirow[t]{10}{*}{ France } & 1 & 0.01 & 100.00 & 0.00 & 0.00 & 44.17 & 55.83 \\
\hline & 2 & 0.01 & 99.88 & 0.12 & 0.01 & 40.11 & 59.89 \\
\hline & 3 & 0.01 & 99.87 & 0.13 & 0.01 & 43.80 & 56.20 \\
\hline & 4 & 0.01 & 99.20 & 0.80 & 0.01 & 46.74 & 53.26 \\
\hline & 5 & 0.01 & 99.15 & 0.85 & 0.01 & 66.66 & 33.34 \\
\hline & 6 & 0.01 & 98.95 & 1.05 & 0.01 & 70.84 & 29.16 \\
\hline & 7 & 0.01 & 98.76 & 1.24 & 0.01 & 70.18 & 29.82 \\
\hline & 8 & 0.01 & 97.87 & 2.13 & 0.01 & 66.92 & 33.08 \\
\hline & 9 & 0.01 & 96.70 & 3.30 & 0.01 & 76.41 & 23.59 \\
\hline & 10 & 0.01 & 96.36 & 3.64 & 0.01 & 75.67 & 24.33 \\
\hline \multirow[t]{10}{*}{ Germany } & 1 & 0.13 & 100.00 & 0.00 & 0.00 & 0.01 & 99.99 \\
\hline & 2 & 0.19 & 95.54 & 4.46 & 0.01 & 10.54 & 89.46 \\
\hline & 3 & 0.21 & 90.19 & 9.81 & 0.01 & 10.00 & 90.00 \\
\hline & 4 & 0.22 & 89.31 & 10.69 & 0.01 & 9.33 & 90.67 \\
\hline & 5 & 0.24 & 88.80 & 11.20 & 0.01 & 8.89 & 91.11 \\
\hline & 6 & 0.25 & 89.11 & 10.89 & 0.01 & 8.96 & 91.04 \\
\hline & 7 & 0.25 & 89.38 & 10.62 & 0.01 & 12.49 & 87.51 \\
\hline & 8 & 0.25 & 89.56 & 10.44 & 0.01 & 22.88 & 77.12 \\
\hline & 9 & 0.26 & 88.08 & 11.92 & 0.01 & 31.42 & 68.58 \\
\hline & 10 & 0.26 & 88.17 & 11.83 & 0.01 & 39.69 & 60.31 \\
\hline \multirow[t]{10}{*}{ Hong Kong, China } & 1 & 0.33 & 100.00 & 0.00 & 0.02 & 0.95 & 99.05 \\
\hline & 2 & 0.45 & 95.35 & 4.65 & 0.03 & 0.60 & 99.40 \\
\hline & 3 & 0.52 & 91.11 & 8.89 & 0.03 & 0.41 & 99.59 \\
\hline & 4 & 0.57 & 87.48 & 12.52 & 0.04 & 0.29 & 99.71 \\
\hline & 5 & 0.61 & 84.20 & 15.80 & 0.04 & 0.23 & 99.77 \\
\hline & 6 & 0.63 & 81.12 & 18.88 & 0.05 & 0.20 & 99.80 \\
\hline & 7 & 0.66 & 78.17 & 21.83 & 0.05 & 0.19 & 99.81 \\
\hline & 8 & 0.68 & 75.34 & 24.66 & 0.06 & 0.20 & 99.80 \\
\hline & 9 & 0.70 & 72.63 & 27.37 & 0.06 & 0.22 & 99.78 \\
\hline & 10 & 0.71 & 70.03 & 29.97 & 0.06 & 0.25 & 99.75 \\
\hline \multirow[t]{10}{*}{ Hungary } & 1 & 0.02 & 100.00 & 0.00 & 0.01 & 38.50 & 61.50 \\
\hline & 2 & 0.02 & 96.41 & 3.59 & 0.01 & 63.90 & 36.10 \\
\hline & 3 & 0.03 & 87.80 & 12.20 & 0.01 & 65.34 & 34.66 \\
\hline & 4 & 0.03 & 86.33 & 13.67 & 0.01 & 65.04 & 34.96 \\
\hline & 5 & 0.03 & 87.32 & 12.68 & 0.01 & 51.08 & 48.92 \\
\hline & 6 & 0.03 & 86.91 & 13.09 & 0.01 & 64.40 & 35.60 \\
\hline & 7 & 0.03 & 78.14 & 21.86 & 0.01 & 66.08 & 33.92 \\
\hline & 8 & 0.03 & 76.04 & 23.96 & 0.01 & 65.23 & 34.77 \\
\hline & 9 & 0.03 & 70.61 & 29.39 & 0.02 & 57.53 & 42.47 \\
\hline & 10 & 0.04 & 72.96 & 27.04 & 0.02 & 63.56 & 36.44 \\
\hline
\end{tabular}


Table 9. Variance decomposition (continued)

\begin{tabular}{|c|c|c|c|c|c|c|c|}
\hline \multirow[t]{2}{*}{ Country } & \multirow[t]{2}{*}{ Period } & \multicolumn{3}{|c|}{$\begin{array}{l}\text { Column (a) } \\
\text { FUTURES }\end{array}$} & \multicolumn{3}{|c|}{$\begin{array}{l}\text { Column (b) } \\
\text { GDP }\end{array}$} \\
\hline & & S.E. & $\begin{array}{c}\varepsilon_{1 \mathrm{t}} \\
\text { (FUTURES) }\end{array}$ & $\begin{array}{c}\varepsilon_{2 t} \\
(\mathrm{GDP})\end{array}$ & S.E. & $\begin{array}{c}\varepsilon_{1 \mathrm{t}} \\
\text { (FUTURES) }\end{array}$ & $\begin{array}{c}\varepsilon_{2 \mathrm{t}} \\
(\mathrm{GDP})\end{array}$ \\
\hline \multirow[t]{10}{*}{ Italy } & 1 & 0.00 & 100.00 & 0.00 & 0.00 & 32.21 & 67.79 \\
\hline & 2 & 0.00 & 99.60 & 0.40 & 0.01 & 37.84 & 62.16 \\
\hline & 3 & 0.00 & 97.77 & 2.23 & 0.01 & 46.67 & 53.33 \\
\hline & 4 & 0.00 & 97.77 & 2.23 & 0.01 & 48.82 & 51.18 \\
\hline & 5 & 0.00 & 79.81 & 20.19 & 0.01 & 47.94 & 52.06 \\
\hline & 6 & 0.00 & 76.64 & 23.36 & 0.01 & 46.21 & 53.79 \\
\hline & 7 & 0.00 & 71.22 & 28.78 & 0.01 & 45.05 & 54.95 \\
\hline & 8 & 0.00 & 70.65 & 29.35 & 0.01 & 43.82 & 56.18 \\
\hline & 9 & 0.00 & 69.87 & 30.13 & 0.01 & 42.78 & 57.22 \\
\hline & 10 & 0.00 & 58.92 & 41.08 & 0.01 & 42.35 & 57.65 \\
\hline \multirow[t]{10}{*}{ Japan } & 1 & 0.01 & 100.00 & 0.00 & 0.01 & 1.65 & 98.35 \\
\hline & 2 & 0.02 & 98.25 & 1.75 & 0.01 & 2.35 & 97.65 \\
\hline & 3 & 0.02 & 97.82 & 2.18 & 0.01 & 5.14 & 94.86 \\
\hline & 4 & 0.02 & 97.77 & 2.23 & 0.02 & 6.21 & 93.79 \\
\hline & 5 & 0.02 & 97.52 & 2.48 & 0.02 & 7.38 & 92.62 \\
\hline & 6 & 0.02 & 96.81 & 3.19 & 0.02 & 9.89 & 90.11 \\
\hline & 7 & 0.02 & 95.39 & 4.61 & 0.02 & 11.58 & 88.42 \\
\hline & 8 & 0.02 & 95.00 & 5.00 & 0.02 & 13.82 & 86.18 \\
\hline & 9 & 0.02 & 94.75 & 5.25 & 0.02 & 14.93 & 85.07 \\
\hline & 10 & 0.03 & 94.59 & 5.41 & 0.02 & 15.23 & 84.77 \\
\hline \multirow[t]{10}{*}{ Netherlands } & 1 & 0.03 & 100.00 & 0.00 & 0.00 & 1.94 & 98.06 \\
\hline & 2 & 0.05 & 93.79 & 6.21 & 0.01 & 5.02 & 94.98 \\
\hline & 3 & 0.06 & 89.85 & 10.15 & 0.01 & 8.51 & 91.49 \\
\hline & 4 & 0.07 & 87.45 & 12.55 & 0.01 & 12.23 & 87.77 \\
\hline & 5 & 0.08 & 85.87 & 14.13 & 0.01 & 15.98 & 84.02 \\
\hline & 6 & 0.08 & 84.74 & 15.26 & 0.02 & 19.62 & 80.38 \\
\hline & 7 & 0.09 & 83.87 & 16.13 & 0.02 & 23.06 & 76.94 \\
\hline & 8 & 0.09 & 83.16 & 16.84 & 0.02 & 26.27 & 73.73 \\
\hline & 9 & 0.10 & 82.57 & 17.43 & 0.02 & 29.24 & 70.76 \\
\hline & 10 & 0.10 & 82.06 & 17.94 & 0.02 & 31.96 & 68.04 \\
\hline \multirow[t]{10}{*}{ New Zealand } & 1 & 0.00 & 100.00 & 0.00 & 0.01 & 13.53 & 86.47 \\
\hline & 2 & 0.00 & 99.99 & 0.01 & 0.01 & 9.39 & 90.61 \\
\hline & 3 & 0.00 & 99.98 & 0.02 & 0.02 & 6.04 & 93.96 \\
\hline & 4 & 0.00 & 99.81 & 0.19 & 0.02 & 10.13 & 89.87 \\
\hline & 5 & 0.00 & 97.23 & 2.77 & 0.02 & 9.17 & 90.83 \\
\hline & 6 & 0.00 & 95.08 & 4.92 & 0.02 & 8.76 & 91.24 \\
\hline & 7 & 0.00 & 94.44 & 5.56 & 0.02 & 12.92 & 87.08 \\
\hline & 8 & 0.00 & 93.29 & 6.71 & 0.02 & 12.59 & 87.41 \\
\hline & 9 & 0.00 & 92.65 & 7.35 & 0.02 & 12.82 & 87.18 \\
\hline & 10 & 0.00 & 91.97 & 8.03 & 0.02 & 12.41 & 87.59 \\
\hline \multirow[t]{10}{*}{ Norway } & 1 & 0.01 & 100.00 & 0.00 & 0.02 & 0.53 & 99.47 \\
\hline & 2 & 0.01 & 92.29 & 7.71 & 0.02 & 0.46 & 99.54 \\
\hline & 3 & 0.01 & 92.31 & 7.69 & 0.02 & 0.69 & 99.31 \\
\hline & 4 & 0.01 & 89.96 & 10.04 & 0.02 & 1.07 & 98.93 \\
\hline & 5 & 0.01 & 89.14 & 10.86 & 0.03 & 1.60 & 98.40 \\
\hline & 6 & 0.01 & 87.68 & 12.32 & 0.03 & 2.08 & 97.92 \\
\hline & 7 & 0.01 & 86.70 & 13.30 & 0.03 & 2.53 & 97.47 \\
\hline & 8 & 0.01 & 85.54 & 14.46 & 0.03 & 2.91 & 97.09 \\
\hline & 9 & 0.01 & 84.56 & 15.44 & 0.03 & 3.24 & 96.76 \\
\hline & 10 & 0.01 & 83.55 & 16.45 & 0.04 & 3.52 & 96.48 \\
\hline
\end{tabular}


Table 9. Variance decomposition (continued)

\begin{tabular}{|c|c|c|c|c|c|c|c|}
\hline \multirow[t]{2}{*}{ Country } & \multirow[t]{2}{*}{ Period } & \multicolumn{3}{|c|}{$\begin{array}{l}\text { Column (a) } \\
\text { FUTURES }\end{array}$} & \multicolumn{3}{|c|}{$\begin{array}{l}\text { Column (b) } \\
\text { GDP }\end{array}$} \\
\hline & & S.E. & $\begin{array}{c}\varepsilon_{1 \mathrm{t}} \\
\text { (FUTURES) }\end{array}$ & $\begin{array}{c}\varepsilon_{2 \mathrm{t}} \\
(\mathrm{GDP})\end{array}$ & S.E. & $\begin{array}{c}\varepsilon_{1 \mathrm{t}} \\
\text { (FUTURES) }\end{array}$ & $\begin{array}{c}\varepsilon_{2 \mathrm{t}} \\
(\mathrm{GDP})\end{array}$ \\
\hline \multirow[t]{10}{*}{ Portugal } & 1 & 0.00 & 100.00 & 0.00 & 0.01 & 18.61 & 81.39 \\
\hline & 2 & 0.01 & 89.60 & 10.40 & 0.01 & 14.44 & 85.56 \\
\hline & 3 & 0.01 & 81.81 & 18.19 & 0.01 & 12.40 & 87.60 \\
\hline & 4 & 0.01 & 78.02 & 21.98 & 0.01 & 15.81 & 84.19 \\
\hline & 5 & 0.01 & 77.12 & 22.88 & 0.01 & 15.92 & 84.08 \\
\hline & 6 & 0.01 & 78.24 & 21.76 & 0.01 & 20.89 & 79.11 \\
\hline & 7 & 0.01 & 78.29 & 21.71 & 0.01 & 26.32 & 73.68 \\
\hline & 8 & 0.01 & 76.21 & 23.79 & 0.01 & 28.48 & 71.52 \\
\hline & 9 & 0.01 & 75.64 & 24.36 & 0.01 & 29.48 & 70.52 \\
\hline & 10 & 0.02 & 76.20 & 23.80 & 0.02 & 32.67 & 67.33 \\
\hline \multirow[t]{10}{*}{ South Africa } & 1 & 0.03 & 100.00 & 0.00 & 0.00 & 3.22 & 96.78 \\
\hline & 2 & 0.03 & 99.33 & 0.67 & 0.01 & 6.51 & 93.49 \\
\hline & 3 & 0.03 & 97.75 & 2.25 & 0.01 & 10.22 & 89.78 \\
\hline & 4 & 0.03 & 95.56 & 4.44 & 0.02 & 13.43 & 86.57 \\
\hline & 5 & 0.03 & 93.06 & 6.94 & 0.02 & 15.99 & 84.01 \\
\hline & 6 & 0.03 & 90.48 & 9.52 & 0.03 & 17.97 & 82.03 \\
\hline & 7 & 0.03 & 87.93 & 12.07 & 0.03 & 19.48 & 80.52 \\
\hline & 8 & 0.03 & 85.49 & 14.51 & 0.03 & 20.64 & 79.36 \\
\hline & 9 & 0.03 & 83.18 & 16.82 & 0.04 & 21.55 & 78.45 \\
\hline & 10 & 0.03 & 81.02 & 18.98 & 0.04 & 22.27 & 77.73 \\
\hline \multirow[t]{10}{*}{ Spain } & 1 & 0.00 & 100.00 & 0.00 & 0.01 & 0.03 & 99.97 \\
\hline & 2 & 0.00 & 99.66 & 0.34 & 0.01 & 1.55 & 98.45 \\
\hline & 3 & 0.00 & 99.20 & 0.80 & 0.01 & 5.78 & 94.22 \\
\hline & 4 & 0.00 & 99.26 & 0.74 & 0.01 & 6.12 & 93.88 \\
\hline & 5 & 0.00 & 98.41 & 1.59 & 0.02 & 6.38 & 93.62 \\
\hline & 6 & 0.00 & 98.36 & 1.64 & 0.02 & 6.52 & 93.48 \\
\hline & 7 & 0.00 & 98.35 & 1.65 & 0.02 & 6.10 & 93.90 \\
\hline & 8 & 0.00 & 98.24 & 1.76 & 0.02 & 6.89 & 93.11 \\
\hline & 9 & 0.00 & 97.52 & 2.48 & 0.02 & 7.04 & 92.96 \\
\hline & 10 & 0.00 & 97.39 & 2.61 & 0.02 & 7.48 & 92.52 \\
\hline \multirow[t]{10}{*}{ Sweden } & 1 & 0.08 & 100.00 & 0.00 & 0.01 & 1.67 & 98.33 \\
\hline & 2 & 0.09 & 97.25 & 2.75 & 0.02 & 5.03 & 94.97 \\
\hline & 3 & 0.11 & 95.63 & 4.37 & 0.02 & 4.78 & 95.22 \\
\hline & 4 & 0.14 & 95.17 & 4.83 & 0.02 & 4.28 & 95.72 \\
\hline & 5 & 0.14 & 91.42 & 8.58 & 0.03 & 3.80 & 96.20 \\
\hline & 6 & 0.15 & 88.88 & 11.12 & 0.03 & 3.55 & 96.45 \\
\hline & 7 & 0.16 & 85.83 & 14.17 & 0.04 & 3.19 & 96.81 \\
\hline & 8 & 0.17 & 82.54 & 17.46 & 0.04 & 2.88 & 97.12 \\
\hline & 9 & 0.17 & 79.81 & 20.19 & 0.04 & 2.51 & 97.49 \\
\hline & 10 & 0.17 & 76.88 & 23.12 & 0.05 & 2.29 & 97.71 \\
\hline \multirow[t]{10}{*}{ Switzerland } & 1 & 0.14 & 100.00 & 0.00 & 0.00 & 0.59 & 99.41 \\
\hline & 2 & 0.19 & 99.49 & 0.51 & 0.01 & 1.87 & 98.13 \\
\hline & 3 & 0.21 & 99.49 & 0.51 & 0.01 & 7.69 & 92.31 \\
\hline & 4 & 0.22 & 99.50 & 0.50 & 0.01 & 16.54 & 83.46 \\
\hline & 5 & 0.23 & 99.14 & 0.86 & 0.01 & 25.51 & 74.49 \\
\hline & 6 & 0.23 & 98.41 & 1.59 & 0.01 & 33.02 & 66.98 \\
\hline & 7 & 0.24 & 97.45 & 2.55 & 0.02 & 38.79 & 61.21 \\
\hline & 8 & 0.24 & 96.43 & 3.57 & 0.02 & 43.10 & 56.90 \\
\hline & 9 & 0.25 & 95.44 & 4.56 & 0.02 & 46.33 & 53.67 \\
\hline & 10 & 0.25 & 94.52 & 5.48 & 0.02 & 48.79 & 51.21 \\
\hline
\end{tabular}


Table 9. Variance decomposition (continued)

\begin{tabular}{cccccccc}
\hline Country & Period & \multicolumn{3}{c}{$\begin{array}{c}\text { Column (a) } \\
\text { FUTURES }\end{array}$} & \multicolumn{3}{c}{$\begin{array}{c}\text { Column (b) } \\
\text { GDP }\end{array}$} \\
\cline { 2 - 7 } & & S.E. & $\begin{array}{c}\varepsilon_{1 \mathrm{t}} \\
\text { (FUTURES) }\end{array}$ & $\begin{array}{c}\varepsilon_{2 \mathrm{t}} \\
(\mathrm{GDP})\end{array}$ & S.E. & $\varepsilon_{1 \mathrm{t}}$ & $\varepsilon_{2 \mathrm{t}}$ \\
(FUTURES) & $(\mathrm{GDP})$ \\
\hline United Kingdom & 1 & 0.06 & 100.00 & 0.00 & 0.01 & 0.03 & 99.97 \\
& 2 & 0.06 & 90.36 & 9.64 & 0.01 & 0.80 & 99.20 \\
& 3 & 0.07 & 85.52 & 14.48 & 0.02 & 0.88 & 99.12 \\
& 4 & 0.08 & 84.84 & 15.16 & 0.02 & 2.21 & 97.79 \\
& 5 & 0.09 & 82.92 & 17.08 & 0.02 & 1.27 & 98.73 \\
& 6 & 0.09 & 82.86 & 17.14 & 0.03 & 0.96 & 99.04 \\
& 7 & 0.10 & 81.74 & 18.26 & 0.03 & 0.82 & 99.18 \\
& 8 & 0.10 & 78.75 & 21.25 & 0.03 & 1.14 & 98.86 \\
& 9 & 0.11 & 75.39 & 24.61 & 0.04 & 0.89 & 99.11 \\
& 10 & 0.11 & 73.34 & 26.66 & 0.04 & 0.89 & 99.11 \\
\hline United States & 1 & 0.02 & 100.00 & 0.00 & 0.00 & 1.81 & 98.19 \\
& 2 & 0.02 & 99.86 & 0.14 & 0.01 & 3.13 & 96.87 \\
& 3 & 0.03 & 99.86 & 0.14 & 0.01 & 4.33 & 95.67 \\
& 4 & 0.03 & 99.71 & 0.29 & 0.01 & 6.10 & 93.90 \\
& 5 & 0.04 & 99.41 & 0.59 & 0.02 & 7.16 & 92.84 \\
& 6 & 0.04 & 98.88 & 1.12 & 0.02 & 7.97 & 92.03 \\
& 7 & 0.04 & 98.35 & 1.65 & 0.02 & 8.37 & 91.63 \\
& 8 & 0.04 & 97.82 & 2.18 & 0.02 & 8.55 & 91.45 \\
& 9 & 0.05 & 97.35 & 2.65 & 0.03 & 8.54 & 91.46 \\
& 10 & 0.05 & 96.92 & 3.08 & 0.03 & 8.43 & 91.57 \\
\hline
\end{tabular}

Variance decompositions of FUTURES and GDP are presented for 22 countries. Variance decomposition breaks down the variance of the forecast error for each variable into components that can be attributed to each of the endogenous variables.

FUTURES : Value of futures contracts divided by GDP.

GDP : Logarithm of Real Gross Domestic Product (seasonalized).

S.E. : Forecast error. 
Table 10. Impulse-response function

\begin{tabular}{|c|c|c|c|c|c|}
\hline \multirow[t]{2}{*}{ Country } & \multirow[t]{2}{*}{$\begin{array}{l}\text { Period } \\
\text { (s) }\end{array}$} & \multicolumn{2}{|c|}{$\begin{array}{c}\text { Column (a) } \\
\text { FUTURES, } \mathrm{y}_{1, \mathrm{t}+\mathrm{s}}\end{array}$} & \multicolumn{2}{|c|}{$\begin{array}{l}\text { Column (b) } \\
\text { GDP, } \mathrm{y}_{2, \mathrm{t}+\mathrm{s}}\end{array}$} \\
\hline & & $\begin{array}{c}\varepsilon_{1 \mathrm{t}} \\
\text { (FUTURES) }\end{array}$ & $\begin{array}{c}\varepsilon_{2 t} \\
(\mathrm{GDP})\end{array}$ & $\begin{array}{c}\varepsilon_{1 \mathrm{t}} \\
(\mathrm{FUTURES})\end{array}$ & $\begin{array}{c}\varepsilon_{2 \mathrm{t}} \\
(\mathrm{GDP}) \\
\end{array}$ \\
\hline \multirow[t]{10}{*}{ Australia } & 1 & 0.005 & 0.000 & -0.001 & 0.004 \\
\hline & 2 & 0.004 & -0.001 & 0.001 & 0.005 \\
\hline & 3 & 0.005 & 0.000 & -0.001 & 0.002 \\
\hline & 4 & 0.006 & -0.002 & -0.001 & 0.001 \\
\hline & 5 & 0.007 & -0.003 & -0.001 & 0.000 \\
\hline & 6 & 0.009 & -0.002 & -0.002 & 0.001 \\
\hline & 7 & 0.012 & -0.002 & -0.002 & 0.004 \\
\hline & 8 & 0.014 & -0.002 & -0.002 & 0.004 \\
\hline & 9 & 0.017 & -0.002 & -0.004 & 0.003 \\
\hline & 10 & 0.022 & -0.004 & -0.005 & 0.002 \\
\hline \multirow[t]{10}{*}{ Austria } & 1 & 0.000 & 0.000 & 0.003 & 0.009 \\
\hline & 2 & 0.000 & 0.000 & -0.001 & 0.007 \\
\hline & 3 & 0.000 & 0.000 & 0.000 & 0.006 \\
\hline & 4 & 0.000 & 0.000 & -0.001 & 0.004 \\
\hline & 5 & 0.000 & 0.000 & 0.000 & 0.004 \\
\hline & 6 & 0.000 & 0.000 & -0.001 & 0.003 \\
\hline & 7 & 0.000 & 0.000 & -0.001 & 0.003 \\
\hline & 8 & 0.000 & 0.000 & 0.000 & 0.002 \\
\hline & 9 & 0.000 & 0.000 & 0.000 & 0.001 \\
\hline & 10 & 0.000 & 0.000 & 0.000 & 0.001 \\
\hline \multirow[t]{10}{*}{ Belgium } & 1 & 0.000 & 0.000 & 0.005 & 0.011 \\
\hline & 2 & 0.000 & 0.000 & 0.001 & 0.011 \\
\hline & 3 & 0.000 & 0.000 & 0.003 & 0.010 \\
\hline & 4 & 0.000 & 0.000 & 0.005 & 0.011 \\
\hline & 5 & 0.000 & 0.000 & 0.006 & 0.011 \\
\hline & 6 & 0.000 & 0.000 & 0.007 & 0.010 \\
\hline & 7 & 0.000 & 0.000 & 0.008 & 0.010 \\
\hline & 8 & 0.000 & 0.000 & 0.009 & 0.010 \\
\hline & 9 & 0.000 & 0.000 & 0.010 & 0.010 \\
\hline & 10 & 0.000 & 0.000 & 0.011 & 0.010 \\
\hline \multirow[t]{10}{*}{ Brazil } & 1 & 0.022 & 0.000 & -0.011 & 0.022 \\
\hline & 2 & 0.021 & 0.000 & 0.004 & -0.008 \\
\hline & 3 & 0.019 & -0.010 & 0.009 & -0.005 \\
\hline & 4 & 0.003 & -0.011 & 0.016 & 0.002 \\
\hline & 5 & 0.008 & -0.010 & 0.000 & 0.008 \\
\hline & 6 & -0.006 & -0.005 & -0.013 & -0.008 \\
\hline & 7 & -0.015 & 0.000 & -0.001 & -0.003 \\
\hline & 8 & -0.022 & 0.003 & 0.006 & 0.000 \\
\hline & 9 & -0.015 & 0.006 & -0.011 & 0.020 \\
\hline & 10 & -0.012 & 0.007 & -0.011 & -0.003 \\
\hline \multirow[t]{10}{*}{ Canada } & 1 & 0.000 & 0.000 & 0.001 & 0.004 \\
\hline & 2 & 0.000 & 0.000 & 0.003 & 0.006 \\
\hline & 3 & 0.001 & 0.000 & 0.001 & 0.005 \\
\hline & 4 & -0.001 & 0.000 & 0.003 & 0.005 \\
\hline & 5 & 0.001 & -0.001 & 0.001 & 0.005 \\
\hline & 6 & -0.001 & 0.001 & 0.002 & -0.001 \\
\hline & 7 & 0.001 & -0.001 & -0.001 & 0.001 \\
\hline & 8 & -0.001 & 0.001 & 0.002 & -0.003 \\
\hline & 9 & 0.000 & -0.002 & 0.000 & 0.001 \\
\hline & 10 & 0.000 & 0.001 & 0.000 & -0.003 \\
\hline
\end{tabular}


Table 10. Impulse-response function (continued)

\begin{tabular}{|c|c|c|c|c|c|}
\hline \multirow[t]{2}{*}{ Country } & \multirow[t]{2}{*}{$\begin{array}{l}\text { Period } \\
\text { (s) }\end{array}$} & \multicolumn{2}{|c|}{$\begin{array}{c}\text { Column (a) } \\
\text { FUTURES, } \mathrm{y}_{1, \mathrm{t}+\mathrm{s}}\end{array}$} & \multicolumn{2}{|c|}{$\begin{array}{l}\text { Column (b) } \\
\text { GDP, } \mathrm{y}_{2, \mathrm{t}+\mathrm{s}}\end{array}$} \\
\hline & & $\begin{array}{c}\varepsilon_{1 \mathrm{t}} \\
\text { (FUTURES) }\end{array}$ & $\begin{array}{c}\varepsilon_{2 \mathrm{t}} \\
(\mathrm{GDP})\end{array}$ & $\begin{array}{c}\varepsilon_{1 \mathrm{t}} \\
(\mathrm{FUTURES})\end{array}$ & $\begin{array}{c}\varepsilon_{2 \mathrm{t}} \\
(\mathrm{GDP}) \\
\end{array}$ \\
\hline \multirow[t]{10}{*}{ Denmark } & 1 & 0.046 & 0.000 & -0.002 & 0.008 \\
\hline & 2 & 0.035 & -0.009 & -0.001 & 0.000 \\
\hline & 3 & 0.031 & -0.009 & 0.002 & 0.002 \\
\hline & 4 & 0.044 & -0.020 & 0.001 & 0.001 \\
\hline & 5 & 0.040 & -0.013 & 0.001 & 0.003 \\
\hline & 6 & 0.035 & -0.014 & 0.001 & 0.000 \\
\hline & 7 & 0.046 & -0.017 & 0.005 & 0.000 \\
\hline & 8 & 0.046 & -0.016 & 0.004 & -0.001 \\
\hline & 9 & 0.032 & -0.010 & 0.000 & 0.002 \\
\hline & 10 & 0.034 & -0.014 & 0.002 & -0.001 \\
\hline \multirow[t]{10}{*}{ France } & 1 & 0.006 & 0.000 & -0.003 & 0.004 \\
\hline & 2 & 0.007 & 0.000 & 0.001 & 0.002 \\
\hline & 3 & 0.004 & 0.000 & 0.002 & -0.001 \\
\hline & 4 & 0.002 & 0.001 & 0.002 & -0.001 \\
\hline & 5 & 0.000 & 0.000 & -0.006 & 0.003 \\
\hline & 6 & -0.002 & 0.000 & -0.005 & 0.002 \\
\hline & 7 & 0.000 & 0.000 & -0.001 & -0.001 \\
\hline & 8 & 0.000 & -0.001 & -0.002 & -0.003 \\
\hline & 9 & 0.002 & -0.001 & -0.008 & 0.002 \\
\hline & 10 & 0.002 & -0.001 & -0.002 & 0.002 \\
\hline \multirow[t]{10}{*}{ Germany } & 1 & 0.128 & 0.000 & 0.000 & 0.005 \\
\hline & 2 & 0.128 & 0.039 & 0.002 & 0.003 \\
\hline & 3 & 0.086 & 0.053 & 0.001 & 0.003 \\
\hline & 4 & 0.063 & 0.030 & 0.000 & 0.002 \\
\hline & 5 & 0.088 & 0.035 & 0.000 & 0.002 \\
\hline & 6 & 0.042 & 0.003 & 0.001 & 0.002 \\
\hline & 7 & 0.044 & 0.007 & 0.002 & 0.001 \\
\hline & 8 & 0.045 & -0.010 & 0.003 & 0.001 \\
\hline & 9 & 0.044 & 0.037 & 0.003 & 0.001 \\
\hline & 10 & 0.037 & 0.011 & 0.004 & 0.002 \\
\hline \multirow[t]{10}{*}{ Hong Kong, China } & 1 & 0.332 & 0.000 & 0.002 & 0.016 \\
\hline & 2 & 0.282 & 0.096 & 0.001 & 0.020 \\
\hline & 3 & 0.236 & 0.121 & 0.001 & 0.021 \\
\hline & 4 & 0.195 & 0.129 & 0.000 & 0.021 \\
\hline & 5 & 0.161 & 0.132 & 0.000 & 0.021 \\
\hline & 6 & 0.133 & 0.134 & -0.001 & 0.021 \\
\hline & 7 & 0.108 & 0.136 & -0.001 & 0.021 \\
\hline & 8 & 0.088 & 0.137 & -0.001 & 0.021 \\
\hline & 9 & 0.071 & 0.138 & -0.001 & 0.021 \\
\hline & 10 & 0.057 & 0.139 & -0.001 & 0.021 \\
\hline \multirow[t]{10}{*}{ Hungary } & 1 & 0.023 & 0.000 & 0.004 & 0.005 \\
\hline & 2 & 0.001 & 0.005 & 0.006 & 0.002 \\
\hline & 3 & 0.006 & 0.008 & 0.002 & 0.000 \\
\hline & 4 & 0.001 & 0.003 & -0.001 & 0.001 \\
\hline & 5 & 0.010 & -0.002 & 0.003 & 0.006 \\
\hline & 6 & -0.010 & -0.004 & 0.007 & 0.001 \\
\hline & 7 & -0.005 & 0.010 & 0.003 & 0.001 \\
\hline & 8 & 0.006 & -0.006 & 0.000 & 0.002 \\
\hline & 9 & 0.000 & -0.009 & 0.005 & 0.006 \\
\hline & 10 & -0.013 & -0.005 & 0.007 & -0.001 \\
\hline
\end{tabular}


Table 10. Impulse-response function (continued)

\begin{tabular}{|c|c|c|c|c|c|}
\hline \multirow[t]{2}{*}{ Country } & \multirow[t]{2}{*}{$\begin{array}{l}\text { Period } \\
\text { (s) }\end{array}$} & \multicolumn{2}{|c|}{$\begin{array}{c}\text { Column (a) } \\
\text { FUTURES, } y_{1, t+s}\end{array}$} & \multicolumn{2}{|c|}{$\begin{array}{l}\text { Column (b) } \\
\text { GDP, } \mathrm{y}_{2, \mathrm{t}+\mathrm{s}}\end{array}$} \\
\hline & & $\begin{array}{c}\varepsilon_{1 \mathrm{t}} \\
\text { (FUTURES) }\end{array}$ & $\begin{array}{c}\varepsilon_{2 t} \\
\text { (GDP) }\end{array}$ & $\begin{array}{c}\varepsilon_{1 \mathrm{t}} \\
(\mathrm{FUTURES})\end{array}$ & $\begin{array}{c}\varepsilon_{2 \mathrm{t}} \\
(\mathrm{GDP}) \\
\end{array}$ \\
\hline \multirow[t]{10}{*}{ Italy } & 1 & 0.000 & 0.000 & -0.003 & 0.004 \\
\hline & 2 & 0.000 & 0.000 & -0.003 & 0.004 \\
\hline & 3 & 0.000 & 0.000 & -0.005 & 0.004 \\
\hline & 4 & 0.000 & 0.000 & -0.004 & 0.003 \\
\hline & 5 & 0.000 & 0.000 & -0.003 & 0.004 \\
\hline & 6 & 0.000 & 0.000 & -0.002 & 0.003 \\
\hline & 7 & 0.000 & 0.000 & -0.001 & 0.003 \\
\hline & 8 & 0.000 & 0.000 & -0.001 & 0.003 \\
\hline & 9 & 0.000 & 0.000 & -0.001 & 0.002 \\
\hline & 10 & 0.000 & 0.000 & 0.000 & 0.001 \\
\hline \multirow[t]{10}{*}{ Japan } & 1 & 0.014 & 0.000 & 0.001 & 0.007 \\
\hline & 2 & 0.011 & -0.002 & 0.001 & 0.008 \\
\hline & 3 & 0.013 & -0.002 & 0.003 & 0.009 \\
\hline & 4 & 0.009 & -0.001 & 0.003 & 0.008 \\
\hline & 5 & 0.004 & -0.001 & 0.003 & 0.008 \\
\hline & 6 & 0.001 & -0.002 & 0.004 & 0.006 \\
\hline & 7 & 0.003 & -0.003 & 0.004 & 0.007 \\
\hline & 8 & 0.002 & -0.002 & 0.004 & 0.006 \\
\hline & 9 & 0.003 & -0.001 & 0.003 & 0.003 \\
\hline & 10 & 0.003 & -0.001 & 0.002 & 0.002 \\
\hline \multirow[t]{10}{*}{ Netherlands } & 1 & 0.034 & 0.000 & 0.001 & 0.005 \\
\hline & 2 & 0.032 & 0.012 & 0.002 & 0.006 \\
\hline & 3 & 0.031 & 0.014 & 0.002 & 0.006 \\
\hline & 4 & 0.030 & 0.015 & 0.003 & 0.006 \\
\hline & 5 & 0.029 & 0.015 & 0.004 & 0.007 \\
\hline & 6 & 0.029 & 0.015 & 0.005 & 0.007 \\
\hline & 7 & 0.028 & 0.015 & 0.005 & 0.007 \\
\hline & 8 & 0.027 & 0.015 & 0.006 & 0.007 \\
\hline & 9 & 0.027 & 0.015 & 0.006 & 0.007 \\
\hline & 10 & 0.026 & 0.014 & 0.007 & 0.007 \\
\hline \multirow[t]{10}{*}{ New Zealand } & 1 & 0.000 & 0.000 & 0.003 & 0.007 \\
\hline & 2 & 0.000 & 0.000 & -0.002 & 0.008 \\
\hline & 3 & 0.000 & 0.000 & 0.001 & 0.010 \\
\hline & 4 & 0.000 & 0.000 & -0.004 & 0.008 \\
\hline & 5 & 0.000 & 0.000 & 0.000 & 0.006 \\
\hline & 6 & 0.000 & 0.000 & -0.001 & 0.005 \\
\hline & 7 & 0.000 & 0.000 & -0.004 & 0.003 \\
\hline & 8 & 0.000 & 0.000 & 0.000 & 0.003 \\
\hline & 9 & 0.000 & 0.000 & -0.002 & 0.003 \\
\hline & 10 & 0.000 & 0.000 & 0.000 & 0.004 \\
\hline \multirow[t]{10}{*}{ Norway } & 1 & 0.008 & 0.000 & 0.001 & 0.016 \\
\hline & 2 & 0.004 & 0.003 & 0.000 & 0.008 \\
\hline & 3 & 0.003 & 0.001 & -0.001 & 0.013 \\
\hline & 4 & 0.001 & 0.002 & -0.002 & 0.010 \\
\hline & 5 & 0.001 & 0.001 & -0.002 & 0.011 \\
\hline & 6 & 0.000 & 0.001 & -0.002 & 0.010 \\
\hline & 7 & 0.000 & 0.001 & -0.003 & 0.011 \\
\hline & 8 & 0.000 & 0.001 & -0.003 & 0.011 \\
\hline & 9 & 0.000 & 0.001 & -0.003 & 0.011 \\
\hline & 10 & 0.000 & 0.001 & -0.003 & 0.011 \\
\hline
\end{tabular}


Table 10. Impulse-response function (continued)

\begin{tabular}{|c|c|c|c|c|c|}
\hline \multirow[t]{2}{*}{ Country } & \multirow[t]{2}{*}{$\begin{array}{l}\text { Period } \\
\text { (s) }\end{array}$} & \multicolumn{2}{|c|}{$\begin{array}{c}\text { Column (a) } \\
\text { FUTURES, } \mathrm{y}_{1, \mathrm{t}+\mathrm{s}}\end{array}$} & \multicolumn{2}{|c|}{$\begin{array}{l}\text { Column (b) } \\
\text { GDP, } \mathrm{y}_{2, \mathrm{t}+\mathrm{s}}\end{array}$} \\
\hline & & $\begin{array}{c}\varepsilon_{1 \mathrm{t}} \\
\text { (FUTURES) }\end{array}$ & $\begin{array}{c}\varepsilon_{2 \mathrm{t}} \\
(\mathrm{GDP})\end{array}$ & $\begin{array}{c}\varepsilon_{1 \mathrm{t}} \\
(\mathrm{FUTURES})\end{array}$ & $\begin{array}{c}\varepsilon_{2 \mathrm{t}} \\
(\mathrm{GDP}) \\
\end{array}$ \\
\hline \multirow[t]{10}{*}{ Portugal } & 1 & 0.005 & 0.000 & -0.004 & 0.008 \\
\hline & 2 & 0.007 & -0.003 & 0.002 & 0.006 \\
\hline & 3 & 0.005 & -0.004 & 0.002 & 0.006 \\
\hline & 4 & 0.004 & -0.003 & 0.003 & 0.003 \\
\hline & 5 & 0.002 & -0.002 & -0.001 & 0.001 \\
\hline & 6 & 0.003 & 0.000 & -0.003 & 0.000 \\
\hline & 7 & 0.005 & -0.002 & -0.004 & 0.002 \\
\hline & 8 & 0.004 & -0.003 & -0.003 & 0.003 \\
\hline & 9 & 0.004 & -0.002 & -0.002 & 0.000 \\
\hline & 10 & 0.003 & -0.001 & -0.003 & 0.001 \\
\hline \multirow[t]{10}{*}{ South Africa } & 1 & 0.025 & 0.000 & 0.001 & 0.005 \\
\hline & 2 & 0.013 & 0.002 & 0.002 & 0.008 \\
\hline & 3 & 0.007 & 0.004 & 0.004 & 0.009 \\
\hline & 4 & 0.004 & 0.005 & 0.005 & 0.011 \\
\hline & 5 & 0.003 & 0.005 & 0.006 & 0.011 \\
\hline & 6 & 0.003 & 0.005 & 0.007 & 0.012 \\
\hline & 7 & 0.003 & 0.006 & 0.007 & 0.012 \\
\hline & 8 & 0.003 & 0.006 & 0.007 & 0.012 \\
\hline & 9 & 0.003 & 0.006 & 0.007 & 0.013 \\
\hline & 10 & 0.003 & 0.006 & 0.008 & 0.013 \\
\hline \multirow[t]{10}{*}{ Spain } & 1 & 0.002 & 0.000 & 0.000 & 0.011 \\
\hline & 2 & 0.001 & 0.000 & -0.001 & 0.001 \\
\hline & 3 & 0.001 & 0.000 & -0.003 & 0.002 \\
\hline & 4 & 0.001 & 0.000 & 0.001 & 0.000 \\
\hline & 5 & 0.000 & 0.000 & 0.003 & 0.010 \\
\hline & 6 & 0.000 & 0.000 & 0.001 & 0.002 \\
\hline & 7 & 0.000 & 0.000 & 0.000 & 0.004 \\
\hline & 8 & 0.000 & 0.000 & 0.001 & 0.000 \\
\hline & 9 & 0.000 & 0.000 & 0.002 & 0.008 \\
\hline & 10 & 0.000 & 0.000 & 0.001 & 0.002 \\
\hline \multirow[t]{10}{*}{ Sweden } & 1 & 0.083 & 0.000 & 0.002 & 0.013 \\
\hline & 2 & 0.041 & 0.016 & 0.004 & 0.011 \\
\hline & 3 & 0.061 & 0.018 & 0.002 & 0.011 \\
\hline & 4 & 0.072 & 0.018 & 0.002 & 0.011 \\
\hline & 5 & 0.037 & 0.030 & 0.003 & 0.017 \\
\hline & 6 & 0.051 & 0.030 & 0.003 & 0.015 \\
\hline & 7 & 0.035 & 0.033 & 0.002 & 0.015 \\
\hline & 8 & 0.017 & 0.033 & 0.001 & 0.014 \\
\hline & 9 & 0.021 & 0.033 & 0.001 & 0.017 \\
\hline & 10 & 0.005 & 0.033 & 0.001 & 0.016 \\
\hline \multirow[t]{10}{*}{ Switzerland } & 1 & 0.145 & 0.000 & 0.000 & 0.005 \\
\hline & 2 & 0.125 & -0.014 & 0.001 & 0.005 \\
\hline & 3 & 0.094 & -0.007 & 0.002 & 0.005 \\
\hline & 4 & 0.067 & 0.005 & 0.004 & 0.005 \\
\hline & 5 & 0.049 & 0.014 & 0.004 & 0.004 \\
\hline & 6 & 0.039 & 0.020 & 0.005 & 0.004 \\
\hline & 7 & 0.035 & 0.024 & 0.005 & 0.004 \\
\hline & 8 & 0.034 & 0.025 & 0.005 & 0.004 \\
\hline & 9 & 0.034 & 0.026 & 0.005 & 0.004 \\
\hline & 10 & 0.035 & 0.026 & 0.005 & 0.004 \\
\hline
\end{tabular}


Table 10. Impulse-response function (continued)

\begin{tabular}{cccccc}
\hline Country & $\begin{array}{c}\text { Period } \\
(\mathrm{s})\end{array}$ & \multicolumn{2}{c}{$\begin{array}{c}\text { Column }(\mathrm{a}) \\
\text { FUTURES, } \mathrm{y}_{1, \mathrm{t}+\mathrm{s}}\end{array}$} & \multicolumn{2}{c}{$\begin{array}{c}\text { Column }(\mathrm{b}) \\
\text { GDP, } \mathrm{y}_{2, \mathrm{t}+\mathrm{s}}\end{array}$} \\
\cline { 3 - 6 } & & $\begin{array}{c}\varepsilon_{1 \mathrm{t}} \\
\text { (FUTURES) }\end{array}$ & $\begin{array}{c}\varepsilon_{2 \mathrm{t}} \\
(\mathrm{GDP})\end{array}$ & $\begin{array}{c}\varepsilon_{1 \mathrm{t}} \\
\text { (FUTURES) }\end{array}$ & $\begin{array}{c}\varepsilon_{2 \mathrm{t}} \\
(\mathrm{GDP})\end{array}$ \\
\hline United Kingdom & 1 & 0.057 & 0.000 & 0.000 & 0.010 \\
& 2 & 0.024 & 0.020 & -0.001 & 0.007 \\
& 3 & 0.028 & 0.019 & -0.001 & 0.009 \\
& 4 & 0.019 & 0.010 & 0.002 & 0.011 \\
& 5 & 0.034 & 0.019 & 0.000 & 0.016 \\
& 6 & 0.023 & 0.011 & 0.000 & 0.014 \\
& 7 & 0.034 & 0.019 & 0.001 & 0.013 \\
& 8 & 0.022 & 0.022 & 0.002 & 0.013 \\
& 9 & 0.011 & 0.023 & 0.000 & 0.018 \\
& 10 & -0.007 & 0.018 & 0.001 & 0.015 \\
\hline United States & 1 & 0.017 & 0.000 & 0.001 & 0.004 \\
& 2 & 0.016 & -0.001 & 0.001 & 0.006 \\
& 3 & 0.016 & -0.001 & 0.002 & 0.008 \\
& 4 & 0.016 & 0.001 & 0.003 & 0.008 \\
& 5 & 0.015 & 0.002 & 0.003 & 0.009 \\
& 6 & 0.015 & 0.003 & 0.003 & 0.009 \\
& 7 & 0.014 & 0.003 & 0.003 & 0.009 \\
& 8 & 0.013 & 0.004 & 0.003 & 0.010 \\
& 9 & 0.013 & 0.004 & 0.003 & 0.010 \\
& 10 & 0.012 & 0.004 & 0.003 & 0.010 \\
\hline
\end{tabular}

Estimates of the impulse-response function analysis are shown. Impulse-response function traces the effect of a one-time shock to one of the endogenous variables on current and future values of itself and the other endogenous variables. One standard deviation of one variable's innovation may affect the other variable negatively, positively, or both, throughout the period. Impulse-response function analysis shows the response of an endogenous variable to the changes in errors of the other variables.

FUTURES : Value of futures contracts divided by GDP.

GDP : Logarithm of Real Gross Domestic Product (seasonalized).

$\varepsilon \quad$ : Innovation. 
Table 11. Variance decomposition for reverse Cholesky ordering

\begin{tabular}{|c|c|c|c|c|c|c|c|}
\hline \multirow[t]{2}{*}{ Country } & \multirow[t]{2}{*}{ Period } & \multicolumn{3}{|c|}{$\begin{array}{l}\text { Column (a) } \\
\text { FUTURES }\end{array}$} & \multicolumn{3}{|c|}{$\begin{array}{l}\text { Column (b) } \\
\text { GDP }\end{array}$} \\
\hline & & S.E. & $\begin{array}{c}\varepsilon_{1 \mathrm{t}} \\
\text { (FUTURES) }\end{array}$ & $\begin{array}{c}\varepsilon_{2 \mathrm{t}} \\
(\mathrm{GDP})\end{array}$ & S.E. & $\begin{array}{c}\varepsilon_{1 \mathrm{t}} \\
\text { (FUTURES) }\end{array}$ & $\begin{array}{c}\varepsilon_{2 \mathrm{t}} \\
(\mathrm{GDP})\end{array}$ \\
\hline \multirow[t]{10}{*}{ Australia } & 1 & 0.00 & 95.50 & 4.50 & 0.00 & 0.00 & 100.00 \\
\hline & 2 & 0.01 & 91.27 & 8.73 & 0.01 & 6.01 & 93.99 \\
\hline & 3 & 0.01 & 91.25 & 8.75 & 0.01 & 5.43 & 94.57 \\
\hline & 4 & 0.01 & 86.11 & 13.89 & 0.01 & 7.31 & 92.69 \\
\hline & 5 & 0.01 & 77.59 & 22.41 & 0.01 & 8.65 & 91.35 \\
\hline & 6 & 0.02 & 78.70 & 21.30 & 0.01 & 14.04 & 85.96 \\
\hline & 7 & 0.02 & 81.61 & 18.39 & 0.01 & 13.87 & 86.13 \\
\hline & 8 & 0.02 & 83.76 & 16.24 & 0.01 & 12.52 & 87.48 \\
\hline & 9 & 0.03 & 85.48 & 14.52 & 0.01 & 17.24 & 82.76 \\
\hline & 10 & 0.04 & 85.27 & 14.73 & 0.01 & 26.11 & 73.89 \\
\hline \multirow[t]{10}{*}{ Austria } & 1 & 0.00 & 91.94 & 8.06 & 0.01 & 0.00 & 100.00 \\
\hline & 2 & 0.00 & 93.18 & 6.82 & 0.01 & 8.58 & 91.42 \\
\hline & 3 & 0.00 & 95.00 & 5.00 & 0.01 & 8.40 & 91.60 \\
\hline & 4 & 0.00 & 93.94 & 6.06 & 0.01 & 9.51 & 90.49 \\
\hline & 5 & 0.00 & 89.02 & 10.98 & 0.01 & 9.95 & 90.05 \\
\hline & 6 & 0.00 & 84.92 & 15.08 & 0.01 & 11.34 & 88.66 \\
\hline & 7 & 0.00 & 78.40 & 21.60 & 0.02 & 11.90 & 88.10 \\
\hline & 8 & 0.00 & 77.80 & 22.20 & 0.02 & 11.95 & 88.05 \\
\hline & 9 & 0.00 & 79.68 & 20.32 & 0.02 & 11.87 & 88.13 \\
\hline & 10 & 0.00 & 80.75 & 19.25 & 0.02 & 11.86 & 88.14 \\
\hline \multirow[t]{10}{*}{ Belgium } & 1 & 0.00 & 81.26 & 18.74 & 0.01 & 0.00 & 100.00 \\
\hline & 2 & 0.00 & 74.91 & 25.09 & 0.02 & 4.59 & 95.41 \\
\hline & 3 & 0.00 & 73.13 & 26.87 & 0.02 & 3.80 & 96.20 \\
\hline & 4 & 0.00 & 72.19 & 27.81 & 0.02 & 2.87 & 97.13 \\
\hline & 5 & 0.00 & 71.47 & 28.53 & 0.03 & 2.33 & 97.67 \\
\hline & 6 & 0.00 & 70.92 & 29.08 & 0.03 & 2.23 & 97.77 \\
\hline & 7 & 0.00 & 70.47 & 29.53 & 0.03 & 2.52 & 97.48 \\
\hline & 8 & 0.00 & 70.09 & 29.91 & 0.03 & 3.14 & 96.86 \\
\hline & 9 & 0.00 & 69.75 & 30.25 & 0.04 & 4.02 & 95.98 \\
\hline & 10 & 0.00 & 69.44 & 30.56 & 0.04 & 5.10 & 94.90 \\
\hline \multirow[t]{10}{*}{ France } & 1 & 0.01 & 55.83 & 44.17 & 0.00 & 0.00 & 100.00 \\
\hline & 2 & 0.01 & 53.19 & 46.81 & 0.01 & 18.32 & 81.68 \\
\hline & 3 & 0.01 & 54.26 & 45.74 & 0.01 & 17.27 & 82.73 \\
\hline & 4 & 0.01 & 55.98 & 44.02 & 0.01 & 15.58 & 84.42 \\
\hline & 5 & 0.01 & 55.99 & 44.01 & 0.01 & 16.88 & 83.12 \\
\hline & 6 & 0.01 & 56.69 & 43.31 & 0.01 & 17.41 & 82.59 \\
\hline & 7 & 0.01 & 56.63 & 43.37 & 0.01 & 19.87 & 80.13 \\
\hline & 8 & 0.01 & 56.37 & 43.63 & 0.01 & 25.20 & 74.80 \\
\hline & 9 & 0.01 & 54.35 & 45.65 & 0.01 & 26.89 & 73.11 \\
\hline & 10 & 0.01 & 53.27 & 46.73 & 0.01 & 25.75 & 74.25 \\
\hline \multirow[t]{10}{*}{ Hungary } & 1 & 0.02 & 61.50 & 38.50 & 0.01 & 0.00 & 100.00 \\
\hline & 2 & 0.02 & 59.61 & 40.39 & 0.01 & 14.42 & 85.58 \\
\hline & 3 & 0.03 & 50.53 & 49.47 & 0.01 & 16.74 & 83.26 \\
\hline & 4 & 0.03 & 49.66 & 50.34 & 0.01 & 20.28 & 79.72 \\
\hline & 5 & 0.03 & 53.81 & 46.19 & 0.01 & 14.46 & 85.54 \\
\hline & 6 & 0.03 & 49.91 & 50.09 & 0.01 & 24.31 & 75.69 \\
\hline & 7 & 0.03 & 53.91 & 46.09 & 0.01 & 25.26 & 74.74 \\
\hline & 8 & 0.03 & 57.18 & 42.82 & 0.01 & 25.55 & 74.45 \\
\hline & 9 & 0.03 & 55.60 & 44.40 & 0.02 & 19.55 & 80.45 \\
\hline & 10 & 0.04 & 51.89 & 48.11 & 0.02 & 27.14 & 72.86 \\
\hline
\end{tabular}


Table 11. Variance decomposition for reverse Cholesky ordering (continued)

\begin{tabular}{|c|c|c|c|c|c|c|c|}
\hline \multirow[t]{2}{*}{ Country } & \multirow[t]{2}{*}{ Period } & \multicolumn{3}{|c|}{$\begin{array}{l}\text { Column (a) } \\
\text { FUTURES }\end{array}$} & \multicolumn{3}{|c|}{$\begin{array}{l}\text { Column (b) } \\
\text { GDP }\end{array}$} \\
\hline & & S.E. & $\begin{array}{c}\varepsilon_{1 \mathrm{t}} \\
\text { (FUTURES) }\end{array}$ & $\begin{array}{c}\varepsilon_{2 \mathrm{t}} \\
(\mathrm{GDP})\end{array}$ & S.E. & $\begin{array}{c}\varepsilon_{1 \mathrm{t}} \\
\text { (FUTURES) }\end{array}$ & $\begin{array}{c}\varepsilon_{2 \mathrm{t}} \\
(\mathrm{GDP})\end{array}$ \\
\hline \multirow[t]{10}{*}{ Italy } & 1 & 0.00 & 67.79 & 32.21 & 0.00 & 0.00 & 100.00 \\
\hline & 2 & 0.00 & 63.86 & 36.14 & 0.01 & 0.60 & 99.40 \\
\hline & 3 & 0.00 & 59.69 & 40.31 & 0.01 & 3.17 & 96.83 \\
\hline & 4 & 0.00 & 59.65 & 40.35 & 0.01 & 3.83 & 96.17 \\
\hline & 5 & 0.00 & 48.78 & 51.22 & 0.01 & 3.43 & 96.57 \\
\hline & 6 & 0.00 & 46.26 & 53.74 & 0.01 & 3.19 & 96.81 \\
\hline & 7 & 0.00 & 40.92 & 59.08 & 0.01 & 3.06 & 96.94 \\
\hline & 8 & 0.00 & 41.88 & 58.12 & 0.01 & 3.00 & 97.00 \\
\hline & 9 & 0.00 & 44.10 & 55.90 & 0.01 & 3.00 & 97.00 \\
\hline & 10 & 0.00 & 31.10 & 68.90 & 0.01 & 3.31 & 96.69 \\
\hline \multirow[t]{10}{*}{ New Zealand } & 1 & 0.00 & 86.47 & 13.53 & 0.01 & 0.00 & 100.00 \\
\hline & 2 & 0.00 & 86.68 & 13.32 & 0.01 & 19.77 & 80.23 \\
\hline & 3 & 0.00 & 86.50 & 13.50 & 0.02 & 13.16 & 86.84 \\
\hline & 4 & 0.00 & 87.15 & 12.85 & 0.02 & 25.04 & 74.96 \\
\hline & 5 & 0.00 & 86.13 & 13.87 & 0.02 & 24.46 & 75.54 \\
\hline & 6 & 0.00 & 82.76 & 17.24 & 0.02 & 24.71 & 75.29 \\
\hline & 7 & 0.00 & 83.93 & 16.07 & 0.02 & 29.50 & 70.50 \\
\hline & 8 & 0.00 & 83.25 & 16.75 & 0.02 & 28.96 & 71.04 \\
\hline & 9 & 0.00 & 81.88 & 18.12 & 0.02 & 29.68 & 70.32 \\
\hline & 10 & 0.00 & 81.97 & 18.03 & 0.02 & 29.05 & 70.95 \\
\hline \multirow[t]{10}{*}{ Portugal } & 1 & 0.00 & 81.39 & 18.61 & 0.01 & 0.00 & 100.00 \\
\hline & 2 & 0.01 & 55.05 & 44.95 & 0.01 & 17.10 & 82.90 \\
\hline & 3 & 0.01 & 45.01 & 54.99 & 0.01 & 23.03 & 76.97 \\
\hline & 4 & 0.01 & 40.45 & 59.55 & 0.01 & 29.98 & 70.02 \\
\hline & 5 & 0.01 & 39.34 & 60.66 & 0.01 & 29.67 & 70.33 \\
\hline & 6 & 0.01 & 41.17 & 58.83 & 0.01 & 32.36 & 67.64 \\
\hline & 7 & 0.01 & 40.42 & 59.58 & 0.01 & 33.19 & 66.81 \\
\hline & 8 & 0.01 & 37.92 & 62.08 & 0.01 & 31.72 & 68.28 \\
\hline & 9 & 0.01 & 36.94 & 63.06 & 0.01 & 32.15 & 67.85 \\
\hline & 10 & 0.02 & 37.38 & 62.62 & 0.02 & 32.94 & 67.06 \\
\hline
\end{tabular}


Table 12. Impulse-response function for reverse Cholesky ordering

\begin{tabular}{|c|c|c|c|c|c|}
\hline \multirow[t]{2}{*}{ Country } & \multirow[t]{2}{*}{$\begin{array}{l}\text { Period } \\
\text { (s) }\end{array}$} & \multicolumn{2}{|c|}{$\begin{array}{c}\text { Column (a) } \\
\text { FUTURES, } \mathrm{y}_{1, \mathrm{t}+\mathrm{s}}\end{array}$} & \multicolumn{2}{|c|}{$\begin{array}{l}\text { Column (b) } \\
\text { GDP, } \mathrm{y}_{2, \mathrm{t}+\mathrm{s}}\end{array}$} \\
\hline & & $\begin{array}{c}\varepsilon_{1 \mathrm{t}} \\
\text { (FUTURES) }\end{array}$ & $\begin{array}{c}\varepsilon_{2 t} \\
(\mathrm{GDP})\end{array}$ & $\begin{array}{c}\varepsilon_{1 \mathrm{t}} \\
(\mathrm{FUTURES})\end{array}$ & $\begin{array}{c}\varepsilon_{2 \mathrm{t}} \\
(\mathrm{GDP}) \\
\end{array}$ \\
\hline \multirow[t]{10}{*}{ Australia } & 1 & 0.005 & -0.001 & 0.000 & 0.005 \\
\hline & 2 & 0.003 & -0.002 & 0.002 & 0.004 \\
\hline & 3 & 0.005 & -0.002 & 0.000 & 0.002 \\
\hline & 4 & 0.006 & -0.003 & -0.001 & 0.001 \\
\hline & 5 & 0.006 & -0.005 & -0.001 & 0.000 \\
\hline & 6 & 0.008 & -0.004 & -0.002 & 0.001 \\
\hline & 7 & 0.011 & -0.004 & -0.002 & 0.004 \\
\hline & 8 & 0.013 & -0.005 & -0.001 & 0.005 \\
\hline & 9 & 0.017 & -0.006 & -0.003 & 0.004 \\
\hline & 10 & 0.021 & -0.009 & -0.004 & 0.003 \\
\hline \multirow[t]{10}{*}{ Austria } & 1 & 0.000 & 0.000 & 0.000 & 0.009 \\
\hline & 2 & 0.000 & 0.000 & -0.003 & 0.006 \\
\hline & 3 & 0.000 & 0.000 & -0.002 & 0.006 \\
\hline & 4 & 0.000 & 0.000 & -0.002 & 0.004 \\
\hline & 5 & 0.000 & 0.000 & -0.002 & 0.004 \\
\hline & 6 & 0.000 & 0.000 & -0.002 & 0.003 \\
\hline & 7 & 0.000 & 0.000 & -0.002 & 0.003 \\
\hline & 8 & 0.000 & 0.000 & -0.001 & 0.002 \\
\hline & 9 & 0.000 & 0.000 & 0.000 & 0.001 \\
\hline & 10 & 0.000 & 0.000 & 0.000 & 0.001 \\
\hline \multirow[t]{10}{*}{ Belgium } & 1 & 0.000 & 0.000 & 0.000 & 0.013 \\
\hline & 2 & 0.000 & 0.000 & -0.004 & 0.011 \\
\hline & 3 & 0.000 & 0.000 & -0.001 & 0.011 \\
\hline & 4 & 0.000 & 0.000 & 0.000 & 0.012 \\
\hline & 5 & 0.000 & 0.000 & 0.001 & 0.012 \\
\hline & 6 & 0.000 & 0.000 & 0.002 & 0.012 \\
\hline & 7 & 0.000 & 0.000 & 0.003 & 0.013 \\
\hline & 8 & 0.000 & 0.000 & 0.003 & 0.013 \\
\hline & 9 & 0.000 & 0.000 & 0.004 & 0.014 \\
\hline & 10 & 0.000 & 0.000 & 0.005 & 0.014 \\
\hline \multirow[t]{10}{*}{ France } & 1 & 0.004 & -0.004 & 0.000 & 0.005 \\
\hline & 2 & 0.005 & -0.005 & 0.002 & 0.001 \\
\hline & 3 & 0.003 & -0.002 & 0.001 & -0.002 \\
\hline & 4 & 0.002 & -0.001 & 0.001 & -0.002 \\
\hline & 5 & 0.000 & 0.000 & -0.003 & 0.006 \\
\hline & 6 & -0.002 & 0.001 & -0.002 & 0.004 \\
\hline & 7 & 0.000 & 0.000 & -0.002 & 0.000 \\
\hline & 8 & -0.001 & -0.001 & -0.003 & -0.001 \\
\hline & 9 & 0.001 & -0.002 & -0.005 & 0.007 \\
\hline & 10 & 0.001 & -0.002 & -0.001 & 0.003 \\
\hline \multirow[t]{10}{*}{ Hungary } & 1 & 0.018 & 0.015 & 0.000 & 0.006 \\
\hline & 2 & -0.002 & 0.004 & 0.003 & 0.005 \\
\hline & 3 & 0.000 & 0.010 & 0.002 & 0.001 \\
\hline & 4 & -0.001 & 0.004 & -0.002 & 0.000 \\
\hline & 5 & 0.009 & 0.004 & -0.001 & 0.007 \\
\hline & 6 & -0.005 & -0.009 & 0.005 & 0.005 \\
\hline & 7 & -0.010 & 0.005 & 0.002 & 0.002 \\
\hline & 8 & 0.009 & -0.001 & -0.001 & 0.001 \\
\hline & 9 & 0.005 & -0.007 & 0.000 & 0.008 \\
\hline & 10 & -0.007 & -0.011 & 0.006 & 0.004 \\
\hline
\end{tabular}


Table 12. Impulse-response function for reverse Cholesky ordering (continued)

\begin{tabular}{|c|c|c|c|c|c|}
\hline \multirow[t]{2}{*}{ Country } & \multirow[t]{2}{*}{$\begin{array}{l}\text { Period } \\
\text { (s) }\end{array}$} & \multicolumn{2}{|c|}{$\begin{array}{c}\text { Column (a) } \\
\text { FUTURES, } \mathrm{y}_{1, \mathrm{t}+\mathrm{s}}\end{array}$} & \multicolumn{2}{|c|}{$\begin{array}{l}\text { Column (b) } \\
\text { GDP, } \mathrm{y}_{2, \mathrm{t}+\mathrm{s}}\end{array}$} \\
\hline & & $\begin{array}{c}\varepsilon_{1 \mathrm{t}} \\
\text { (FUTURES) }\end{array}$ & $\begin{array}{c}\varepsilon_{2 \mathrm{t}} \\
(\mathrm{GDP})\end{array}$ & $\begin{array}{c}\varepsilon_{1 \mathrm{t}} \\
\text { (FUTURES) }\end{array}$ & $\begin{array}{c}\varepsilon_{2 \mathrm{t}} \\
(\mathrm{GDP})\end{array}$ \\
\hline \multirow[t]{10}{*}{ Italy } & 1 & 0.000 & 0.000 & 0.000 & 0.005 \\
\hline & 2 & 0.000 & 0.000 & -0.001 & 0.005 \\
\hline & 3 & 0.000 & 0.000 & -0.002 & 0.006 \\
\hline & 4 & 0.000 & 0.000 & -0.001 & 0.005 \\
\hline & 5 & 0.000 & 0.000 & -0.001 & 0.005 \\
\hline & 6 & 0.000 & 0.000 & 0.000 & 0.003 \\
\hline & 7 & 0.000 & 0.000 & 0.000 & 0.003 \\
\hline & 8 & 0.000 & 0.000 & 0.000 & 0.003 \\
\hline & 9 & 0.000 & 0.000 & 0.000 & 0.003 \\
\hline & 10 & 0.000 & 0.000 & 0.001 & 0.001 \\
\hline \multirow[t]{10}{*}{ New Zealand } & 1 & 0.000 & 0.000 & 0.000 & 0.007 \\
\hline & 2 & 0.000 & 0.000 & -0.005 & 0.007 \\
\hline & 3 & 0.000 & 0.000 & -0.002 & 0.010 \\
\hline & 4 & 0.000 & 0.000 & -0.007 & 0.006 \\
\hline & 5 & 0.000 & 0.000 & -0.003 & 0.005 \\
\hline & 6 & 0.000 & 0.000 & -0.003 & 0.004 \\
\hline & 7 & 0.000 & 0.000 & -0.005 & 0.001 \\
\hline & 8 & 0.000 & 0.000 & -0.001 & 0.003 \\
\hline & 9 & 0.000 & 0.000 & -0.002 & 0.002 \\
\hline & 10 & 0.000 & 0.000 & -0.001 & 0.004 \\
\hline \multirow[t]{10}{*}{ Portugal } & 1 & 0.004 & -0.002 & 0.000 & 0.008 \\
\hline & 2 & 0.005 & -0.006 & 0.004 & 0.005 \\
\hline & 3 & 0.003 & -0.005 & 0.004 & 0.005 \\
\hline & 4 & 0.002 & -0.004 & 0.004 & 0.002 \\
\hline & 5 & 0.001 & -0.002 & 0.000 & 0.001 \\
\hline & 6 & 0.002 & -0.001 & -0.003 & 0.002 \\
\hline & 7 & 0.003 & -0.004 & -0.003 & 0.003 \\
\hline & 8 & 0.002 & -0.004 & -0.002 & 0.004 \\
\hline & 9 & 0.002 & -0.004 & -0.001 & 0.001 \\
\hline & 10 & 0.002 & -0.003 & -0.002 & 0.003 \\
\hline
\end{tabular}

